UCDPHY-PUB-95-26

IP-ASTP-13-95

January, 1996

\title{
Analysis of Two-Body Decays of Charmed Baryons Using the Quark-Diagram Scheme
}

\author{
Ling-Lie Chau \\ Physics Department, University of California at Davis, California 95616 \\ Hai-Yang Cheng and B. Tseng \\ Institute of Physics, Academia Sinica, Taipei, Taiwan 11529
}

\begin{abstract}
We give a general formulation of the quark-diagram scheme for the nonleptonic weak decays of baryons. We apply it to all the decays of the antitriplet and sextet charmed baryons and express their decay amplitudes in terms of the quark-diagram amplitudes. We have also given parametrizations for the effects of final-state interactions. For SU(3) violation effects, we only parametrize those in the horizontal $W$-loop quark diagrams whose contributions are solely due to $\mathrm{SU}(3)$-violation effects. In the absence of all these effects, there are many relations among various decay modes. Some of the relations are valid even in the presence of final-state interactions when each decay amplitude in the relation contains only a single phase shift. All these relations provide useful frameworks to compare with future experiments and to find out the effects of final-state interactions and SU(3) symmetry violations.
\end{abstract}




\section{Introduction}

The study of charmed baryon physics is of current interest [1]. Many nonleptonic weak decay modes of the charmed baryons $\Lambda_{c}^{+}, \Xi_{c}^{0 A}$ and $\Xi_{c}^{+A}$ have been measured [2] and more data are expected in the near future. Apart from model calculations [3-5], it is useful to study the nonleptonic weak decays in a way which is as model independent as possible. The two-body nonleptonic decays of charmed baryons have been analyzed in terms of SU(3)irreducible-representation (SU(3)-IR) amplitudes [6,7]. However, the quark-diagram scheme (i.e., analyzing the decays in terms of quark-diagram amplitudes) has the advantage that it is more intuitive and easier for implementing model calculations. It has been successfully applied to the hadronic weak decays of charmed and bottom mesons $[8,9]$. It has provided a framework with which we not only can do the least-model-dependent data analysis and give predictions but also make evaluations of theoretical model calculations. In this paper we give a general and unified formulation (always using the orthonormal quark states and independent of $\mathrm{SU}(6)$ symmetry) of the quark-diagram scheme for the nonleptonic weak decays of baryons, which can be useful for all baryon (charm and bottom) nonleptonic decays. Here we apply it to all the two-body hadronic decays (quark-mixing allowed, suppressed, and doubly-suppressed) of the antitriplet and sextet charmed baryons and express them in terms of the quark-diagram amplitudes. We find consistent comparisons with the SU(3)-IR results of Ref. [6]. In addition, the symmetry properties of the initial and final baryon wave functions in conjunction with the Pati-Woo theorem [11] for weak decays enable us to obtain more specific results than those from the SU(3)-IR scheme.

We have also given parametrizations for effects of final-state interactions. For SU(3) violations we indicate only those arising in the horizontal $W$-loop quark diagrams whose contributions are solely due to $\mathrm{SU}(3)$ violation effects. (See more detailed discussion about this at the end of Section III.a.) In the absence of these effects, there are many relations among various decay modes. Some of the relations are valid even in the presence of final-state interactions when each decay amplitude in the relation contains only a single phase shift. It will be interesting to compare these relations with future experimental data. They provide a useful framework to find out the effects of final-state interactions and $\mathrm{SU}(3)$ violations.

Earlier Kohara had given a quark-diagram formulation for the quark-mixing-allowed decays of the antitriplet charmed baryons [10], with which our results agree. However, for the decay products containing an octet baryon he used a basis of quark states which is not orthonormal (which is all right though more complicated) and its choice seems to be based upon exact $\mathrm{SU}(6)$ symmetry (which is not all right since $\mathrm{SU}(6)$ is not an exact symmetry). The fact that our formulation demonstrates that the quark diagram results are independent of whether one uses exact $\mathrm{SU}(6)$ or not helps to clarify this point for his formulation and 
explain why our results can possibly agree. (For detailed comments see Sections II after Eq. (28) and for exact relations between amplitudes see Eq. (63) in Section IV.b.)

In the framework of the quark-diagram scheme, all nonleptonic meson decays can be expressed in terms of six quark-diagram amplitudes [8]: $\mathcal{A}$, the external $W$-emission diagram; $\mathcal{B}$, the internal $W$-emission diagram; $\mathcal{C}$, the $W$-exchange diagram; $\mathcal{D}$, the $W$-annihilation diagram; $\mathcal{E}$, the horizontal $W$-loop diagram; and $\mathcal{F}$, the vertical $W$-loop diagram. These quark diagrams are specific and well-defined physical quantities. They are classified according to the topology of first-order weak interactions, with all QCD strong-interaction effects included. It is important to emphasize that strong interactions do not alter the classification of these diagrams. These quark diagrams have a one-to-one correspondence to those amplitudes classified according to SU(3) irreducible representations.

For the baryon decays, we can easily show by diagram drawing that the $\mathcal{D}$ and $\mathcal{F}$ types of amplitudes do not contribute. However, there are more possibilities in drawing the $\mathcal{C}$ and $\mathcal{E}$ types of amplitudes. More importantly, baryons being made out of three quarks, in contrast to two quarks for the mesons, bring along many essential complications. Though many textbooks [12] have discussed the baryon wave functions, we need to carefully develop the proper formulation suitable for the construction of the quark diagram scheme for the baryon decays. This is what we discuss in Section II, where the relations between the quark states and the baryon states are derived. We then apply this general results to the specific decays of the charmed baryons. In Sections III and IV we give the quark-diagram formulation for the two-body decays of antitriplet charmed baryons into a pseudoscalar meson and a baryon (decuplet and octet); first for the case without effects of final-state interactions and $\mathrm{SU}(3)$ violations, and then for the case with these effects. We discuss their experimental implications and comment on previous related theoretical work. Section V is devoted to studying the nonleptonic weak decays of sextet charmed baryons. In Section VI we give a few concluding remarks.

\section{Quark States and Particle States}

To develop a quark diagram scheme we need to fully understand the relation between the quark states and the particle states. Baryons are made out of three $\frac{1}{2}$-spin quarks. The baryon states form irreducible representations of SU(3)-flavor and SU(2)-spin from the tensor-product states of flavor and spin of three quarks which are written as the following orthonormalized states:

$$
\left|q_{1}, S_{1 z} ; q_{2}, S_{2 z} ; q_{3}, S_{3 z}\right\rangle=\left|q_{1} q_{2} q_{3}\right\rangle\left|S_{1 z} S_{2 z} S_{3 z}\right\rangle
$$

There are $3 \times 3 \times 3=27$ flavor states $\left|q_{1} q_{2} q_{3}\right\rangle$ and $2 \times 2 \times 2=8$ spin states $\left|S_{1 z} S_{2 z} S_{3 z}\right\rangle$. 
Let us first discuss the flavor irreducible representation states of the three quarks. The 27 tri-quark states can be decomposed into $[8]_{A},[8]_{S},[1]_{A}$, and $[10]_{S_{t}}$ irreducible representations, denoted by the orthonormalized states:

$$
\left|\psi(8)_{A}\right\rangle,\left|\psi(8)_{S}\right\rangle,\left|\psi(1)_{A}\right\rangle, \text { and }\left|\psi(10)_{S_{t}}\right\rangle \text {. }
$$

The transformation between the two bases, Eq. (1) and (2), can be written in a $27 \times 27$ matrix which is block-diagonalized into the following sub-matrix transformations:

$$
\left(\begin{array}{c}
\left|\psi^{k}(8)_{S}\right\rangle \\
\left|\psi^{k}(8)_{A}\right\rangle \\
\left|\psi^{k}(10)_{S_{t}}\right\rangle
\end{array}\right)=\left(\begin{array}{ccc}
\frac{1}{\sqrt{6}} & \frac{1}{\sqrt{6}} & -\frac{2}{\sqrt{6}} \\
\frac{1}{\sqrt{2}} & -\frac{1}{\sqrt{2}} & 0 \\
\frac{1}{\sqrt{3}} & \frac{1}{\sqrt{3}} & \frac{1}{\sqrt{3}}
\end{array}\right) \quad\left(\begin{array}{ccc}
\mid q_{a} & \left.q_{b} q_{a}\right\rangle \\
\mid q_{b} & q_{a} & \left.q_{a}\right\rangle \\
\mid q_{a} & q_{a} & \left.q_{b}\right\rangle
\end{array}\right),
$$

where $k$ can be the proton, neutron, $\Sigma^{+}, \Sigma^{-}, \Xi^{0}, \Xi^{-}$types and all of which have two identical quarks. There are 6 of such $3 \times 3$ matrix equations totalling the transformations of the 18 states out of the 27 . Note that the subscripts $A$ and $S$ signify the antisymmetry and symmetry, respectively, between the first two quarks; the subscript $S_{t}$ denotes the total symmetry among the three quarks. Then there are the following transformations of the 6 states with all three quarks being different:

$$
\left(\begin{array}{c}
\left|\psi^{\Sigma}(8)_{S}\right\rangle \\
\left|\psi^{\Sigma}(8)_{A}\right\rangle \\
\left|\psi^{\Lambda}(8)_{S}\right\rangle \\
\left|\psi^{\Lambda}(8)_{A}\right\rangle \\
\left|\psi^{\Lambda_{1}}(1)_{A}\right\rangle \\
\left|\psi^{\Sigma}(10)_{S_{t}}\right\rangle
\end{array}\right)=\left(\begin{array}{cccccc}
\frac{1}{\sqrt{12}} & \frac{1}{\sqrt{12}} & \frac{1}{\sqrt{12}} & \frac{1}{\sqrt{12}} & \frac{-2}{\sqrt{12}} & \frac{-2}{\sqrt{12}} \\
\frac{-1}{2} & \frac{1}{2} & \frac{-1}{2} & \frac{1}{2} & 0 & 0 \\
\frac{1}{2} & \frac{1}{2} & \frac{-1}{2} & \frac{-1}{2} & 0 & 0 \\
\frac{1}{\sqrt{12}} & \frac{-1}{\sqrt{12}} & \frac{-1}{\sqrt{12}} & \frac{1}{\sqrt{12}} & \frac{-2}{\sqrt{12}} & \frac{2}{\sqrt{12}} \\
\frac{1}{\sqrt{6}} & \frac{-1}{\sqrt{6}} & \frac{1}{\sqrt{6}} & \frac{-1}{\sqrt{6}} & \frac{1}{\sqrt{6}} & \frac{-1}{\sqrt{6}} \\
\frac{1}{\sqrt{6}} & \frac{1}{\sqrt{6}} & \frac{1}{\sqrt{6}} & \frac{1}{\sqrt{6}} & \frac{1}{\sqrt{6}} & \frac{1}{\sqrt{6}}
\end{array}\right) \quad\left(\begin{array}{l}
|s d u\rangle \\
|d s u\rangle \\
|s u d\rangle \\
|u s d\rangle \\
|d u s\rangle \\
|u d s\rangle
\end{array}\right)
$$

Finally, there are the three states with all three identical quarks:

$$
\begin{aligned}
\left|\Delta^{++}\right\rangle & =|u u u\rangle \\
\left|\Delta^{-}\right\rangle & =|d d d\rangle \\
\left|\Omega^{-}\right\rangle & =|s s s\rangle .
\end{aligned}
$$

They give three diagonal transformations. These 27 equations, Eqs. (3) to (7), are actually equivalent to the following 27 equations:

$$
\begin{aligned}
\left|\psi^{k}(8)_{A}\right\rangle & =\sum_{q_{i}=u, s, d}\left|q_{1} q_{2} q_{3}\right\rangle\left\langle q_{1} q_{2} q_{3} \mid \psi^{k}(8)_{A}\right\rangle, \\
\left|\psi^{k}(8)_{S}\right\rangle & =\sum_{q_{i}=u, s, d}\left|q_{1} q_{2} q_{3}\right\rangle\left\langle q_{1} q_{2} q_{3} \mid \psi^{k}(8)_{S}\right\rangle, \\
\left|\psi^{k}(1)_{A}\right\rangle & =\sum_{q_{i}=u, s, d}\left|q_{1} q_{2} q_{3}\right\rangle\left\langle q_{1} q_{2} q_{3} \mid \psi^{k}(1)_{A}\right\rangle, \\
\left|\psi^{k}(10)_{S_{t}}\right\rangle & =\sum_{q_{i}=u, s, d}\left|q_{1} q_{2} q_{3}\right\rangle\left\langle q_{1} q_{2} q_{3} \mid \psi^{k}(10)_{S_{t}}\right\rangle,
\end{aligned}
$$


where the superscript $k$ stands for the particles in the multiplets, respectively. These equations are obtained simply by multiplying the left-hand side (l.h.s.) of these equations by the identity operator

$$
\hat{I}=\sum_{q_{i}=u, d, s}\left|q_{1} q_{2} q_{3}\right\rangle\left\langle q_{1} q_{2} q_{3}\right|
$$

which is the completeness of the orthonormal $\left|q_{1} q_{2} q_{3}\right\rangle$-basis in the tri-quark vector space. $\left\langle q_{1} q_{2} q_{3} \mid \psi^{k}(\cdots)\right\rangle$ numbers in Eqs. (8) to (11) are precisely those matrix elements in Eqs. (3) to $(7)$.

Since the transformations, Eqs. (3) to (7), are between two sets of orthonormal bases, the transformation matrix formed by these elements $\left\langle q_{1} q_{2} q_{3} \mid \psi^{k}(\cdots)\right\rangle$ is orthogonal. We can easily take the inverse of the transformation (i.e. taking the transpose of the matrix) and express the quark states in terms of the irreducible representation states, i.e., the particle states. If the basis vectors are not orthonormal, not only such inverse will be harder to find but also the completeness equation will be more complicated than that given by Eq. (12). Therefore, whenever possible, it is better to use the orthonormal basis.

Alternatively, we can also use the basis composed of the quark states that are symmetric and antisymmetric in the first two quarks, i.e.,

$$
\begin{aligned}
\left|\left\{q_{a} q_{b}\right\} q_{c}\right\rangle & \equiv \frac{1}{\sqrt{2}\left(1-\delta_{a b}\right)+2 \delta_{a b}}\left(\left|q_{a} q_{b} q_{c}\right\rangle+\left|q_{b} q_{a} q_{c}\right\rangle\right) \\
\left|\left[q_{a} q_{b}\right] q_{c}\right\rangle & \equiv \frac{1}{\sqrt{2}}\left(\left|q_{a} q_{b} q_{c}\right\rangle-\left|q_{b} q_{a} q_{c}\right\rangle\right)
\end{aligned}
$$

or inversely,

$$
\left|q_{a} q_{b} q_{c}\right\rangle=\frac{\sqrt{2}\left(1-\delta_{a b}\right)+2 \delta_{a b}}{2}\left(\left|\left\{q_{a} q_{b}\right\} q_{c}\right\rangle+\left|\left[q_{a} q_{b}\right] q_{c}\right\rangle\right) .
$$

In this basis, Eqs. (3) and (4) become

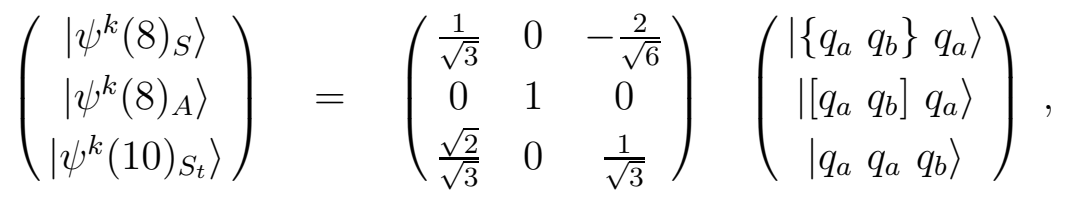

and

$$
\left(\begin{array}{c}
\left|\psi^{\Sigma}(8)_{S}\right\rangle \\
\left|\psi^{\Sigma}(8)_{A}\right\rangle \\
\left|\psi^{\Lambda}(8)_{S}\right\rangle \\
\left|\psi^{\Lambda}(8)_{A}\right\rangle \\
\left|\psi^{\Lambda_{1}}(1)_{A}\right\rangle \\
\left|\psi^{\Sigma}(10)_{S_{t}}\right\rangle
\end{array}\right)=\left(\begin{array}{cccccc}
\frac{1}{\sqrt{6}} & 0 & \frac{1}{\sqrt{6}} & 0 & \frac{-2}{\sqrt{6}} & 0 \\
0 & \frac{-1}{\sqrt{2}} & 0 & \frac{-1}{\sqrt{2}} & 0 & 0 \\
\frac{1}{\sqrt{2}} & 0 & \frac{-1}{\sqrt{2}} & 0 & 0 & 0 \\
0 & \frac{1}{\sqrt{6}} & 0 & \frac{-1}{\sqrt{6}} & 0 & \frac{-2}{\sqrt{6}} \\
0 & \frac{1}{\sqrt{3}} & 0 & \frac{1}{\sqrt{3}} & 0 & \frac{1}{\sqrt{3}} \\
\frac{1}{\sqrt{3}} & 0 & \frac{1}{\sqrt{3}} & 0 & \frac{1}{\sqrt{3}} & 0
\end{array}\right) \quad\left(\begin{array}{c}
|\{s d\} u\rangle \\
|[s d] u\rangle \\
|\{s u\} d\rangle \\
|[s u] d\rangle \\
|\{d u\} s\rangle \\
|[d u] s\rangle
\end{array}\right)
$$


Likewise, in this basis the identity matrix becomes

$$
\hat{I}=\sum_{q_{a}, q_{b}, q_{c}}\left(\left|\left\{q_{a} q_{b}\right\} q_{c}\right\rangle\left\langle\left\{q_{a} q_{b}\right\} q_{c}|+|\left[q_{a} q_{b}\right] q_{c}\right\rangle\left\langle\left[\begin{array}{ll}
q_{a} & q_{b}
\end{array}\right] q_{c}\right|\right) .
$$

Then Eqs.(8)-(11) can be recast into the following form:

$$
\begin{aligned}
\left|\psi^{k}(8)_{A}\right\rangle & =\sum_{q_{a}, q_{b}, q_{c}}\left|\left[\begin{array}{ll}
q_{a} & q_{b}
\end{array}\right] q_{c}\right\rangle\left\langle\left[\begin{array}{ll}
q_{a} & q_{b}
\end{array}\right] q_{c} \mid \psi^{k}(8)_{A}\right\rangle \\
\left|\psi^{k}(8)_{S}\right\rangle & =\sum_{q_{a}, q_{b}, q_{c}}\left|\left\{\begin{array}{ll}
q_{a} & q_{b}
\end{array}\right\} q_{c}\right\rangle\left\langle\left\{\begin{array}{ll}
q_{a} & q_{b}
\end{array}\right\} q_{c} \mid \psi^{k}(8)_{S}\right\rangle, \\
\left|\psi^{k}(1)_{A}\right\rangle & =\sum_{q_{a}, q_{b}, q_{c}}\left|\left[\begin{array}{lll}
q_{a} & q_{b}
\end{array}\right] q_{c}\right\rangle\left\langle\left[\begin{array}{lll}
q_{a} & q_{b}
\end{array}\right] q_{c} \mid \psi^{k}(1)_{A}\right\rangle, \\
\left|\psi^{k}(10)_{S_{t}}\right\rangle & =\sum_{q_{a}, q_{b}, q_{c}}\left|\left\{\begin{array}{lll}
q_{a} & q_{b}
\end{array}\right\} q_{c}\right\rangle\left\langle\left\{\begin{array}{lll}
q_{a} & q_{b}
\end{array} q_{c}\left|\psi^{k}(10)_{S_{t}}\right\rangle\right.\right.
\end{aligned}
$$

where we have used $\left\langle\left[\begin{array}{ll}q_{a} & q_{b}\end{array}\right] q_{c} \mid \psi^{k}(8)_{S}\right\rangle=0$ and $\left\langle\left\{q_{a} q_{b}\right\} q_{c} \mid \psi^{k}(8)_{A}\right\rangle=0$. The coefficients on the right-hand side (r.h.s.) of Eqs. (19)-(22) are the matrix elements in Eqs. (16) and (17).

Here we would like to emphasize that it is conceptually and practically simpler to consistently use the orthonormal quark states as the basis so that the identity operator has the simple expressions of Eq. (12) or Eq. (18). They provide the proper transformation from the particle states to the quark states and vice versa as given by Eqs. (3) and (4), equivalently by Eq. (8) to (11); or Eqs. (16) and (17), equivalently Eqs. (19) to (22). These are the crucial relations we shall use in converting decay amplitudes in terms of particles to decay amplitudes in terms of quarks, i.e., the quark diagram amplitudes.

Next we can form irreducible representations for the spin part of the particle from the tri- $\frac{1}{2}$-spin states

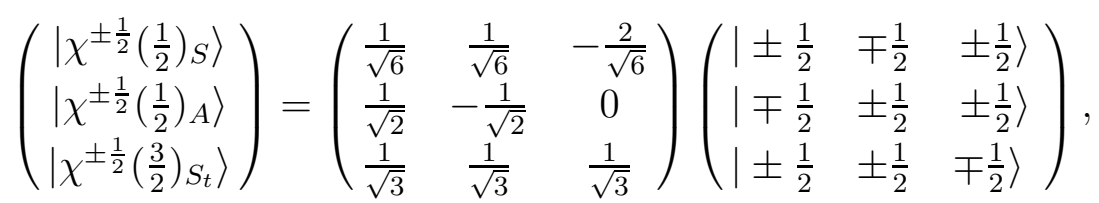

giving 6 equations; and

$$
\left|\chi^{ \pm \frac{3}{2}}\left(\frac{3}{2}\right)_{S_{t}}\right\rangle=\left| \pm \frac{1}{2} \pm \frac{1}{2} \pm \frac{1}{2}\right\rangle
$$

giving 2 diagonal ones; totalling 8 equations. The inverse of these equations is also easy to write out.

The baryon states must be totally antisymmetric in interchanging the composing quarks. Since the color part (which we do not discuss here, see e.g., Ref. [12]) is antisymmetric, the product of the flavor and the spin parts must be symmetric as the spatial wave function is symmetric for low-lying baryons. The decuplet baryons are made out of

$$
\left|B^{m, k}(10)\right\rangle=\left|\chi^{m}\left(\frac{3}{2}\right)_{S_{t}}\right\rangle\left|\psi^{k}(10)_{S_{t}}\right\rangle, \quad m= \pm \frac{1}{2}, \pm \frac{3}{2}, \text { and } k=1 \text { to } 10 .
$$


The octet baryon is a combination of two parts

$$
\begin{aligned}
\left|B^{m, k}(8)\right\rangle & =a\left|B_{A}^{m, k}(8)\right\rangle+b\left|B_{S}^{m, k}(8)\right\rangle \\
& =a\left|\chi^{m}\left(\frac{1}{2}\right)_{A}\right\rangle\left|\psi^{k}(8)_{A}\right\rangle+b\left|\chi^{m}\left(\frac{1}{2}\right)_{S}\right\rangle\left|\psi^{k}(8)_{S}\right\rangle
\end{aligned}
$$

where

$$
|a|^{2}+|b|^{2}=1
$$

The precise values of $a$ and $b$ are not known. Importantly, our formalism does not need such information. We clearly show that the quark-diagram scheme is independent of the values of $a$ and $b$.

If one assumes the $\mathrm{SU}(6)$ symmetry, then $a=b=\frac{1}{\sqrt{2}}$ and the octet baryon wave function can be rewritten as

$$
\left|B^{m, k}(8)\right\rangle=\frac{\sqrt{2}}{3}\left(\left|\chi_{A_{12}}^{m}\left(\frac{1}{2}\right)\right\rangle\left|\psi_{A_{12}(8)}^{k}\right\rangle+\left|\chi_{A_{13}}^{m}\left(\frac{1}{2}\right)\right\rangle\left|\psi_{A_{13}(8)}^{k}\right\rangle+\left|\chi_{A_{23}}^{m}\left(\frac{1}{2}\right)\right\rangle\left|\psi_{A_{23}(8)}^{k}\right\rangle\right),
$$

where the subscripts $i j$ to $A$ and $S$ indicate the pair that is antisymmetric or symmetric. However, $\mathrm{SU}(6)$ is not an exact symmetry. It is important to show that the quark-diagram scheme does not depend on it. Our formulation indeed confirms this requirement.

Earlier Kohara had given a quark-diagram formulation for the quark-mixing-allowed decays of the antitriplet charmed baryons [10]. For $B_{c}(\overline{3}) \rightarrow B(8)+M(8)$ decays (of which he considered only the quark-mixing-allowed decays), it seems that Kohara [10] was motivated by Eq. (28), which is true only if SU(6) symmetry is exact, and chose the octet baryon state to be an equal combination of two different pairs of quarks both antisymmetric in flavor and spin, i.e. two of the terms in Eq. (28), which one can easily check that the two terms are not orthonormal and they are not the conventional way of making the octet baryon wave function. Choosing non-orthonormal basis for the quark states, if done correctly, is all right though complicated. [Kohara's paper did not provide enough detailed information in his paper for us to check directly his calculations without redoing all his formulation in the nonorthonormal basis he used. However, we can compare our results with his for the part he had calculated. Indeed our results agree (see detailed comparisons given later by Eq. (63).] However, we would still like to emphasize that the use of orthonormal basis is much more convenient and, very importantly, that we have made sure that the quark-diagram scheme does not depend upon SU(6) symmetry at all. For the $B_{c}(\overline{3}) \rightarrow B(10)+M(8)$ decays (of which Kohara also only considered the mixing-matrix-allowed decays), Kohara used the same basis as we use and our results agree. 
Besides the $\left|B^{m, k}(8)\right\rangle$ states as given by Eq. (26), there are the states orthogonal to them, which are denoted by

$$
\left|B_{\perp}^{m, k}(8)\right\rangle=b^{*}\left|\chi^{m}\left(\frac{1}{2}\right)_{A}\right\rangle\left|\psi^{k}(8)_{A}\right\rangle-a^{*}\left|\chi^{m}\left(\frac{1}{2}\right)_{S}\right\rangle\left|\psi^{k}(8)_{S}\right\rangle
$$

and $\left\langle B_{\perp}^{m, k}(8) \mid B^{m, k}(8)\right\rangle=0$. Nature does not realize these states, but they are there in the formalism and hence must be considered when completeness of these states is used.

Likewise, we can formulate the meson case, which is much simpler than the baryon case. We discuss it here for completeness and for comparison. Mesons are made out of $\frac{1}{2}$-spin quark-antiquark $q^{\prime} \bar{q}$ pair belonging to the flavor $[3] \times[\overline{3}]$ representation. They form flavor irreducible representations of the $3 \times \overline{3}=9=8+1$, i.e., the 9 quark-antiquark states can be decomposed into flavor [8] and [1] irreducible states denoted by $\left|\phi^{j}(8)\right\rangle$ and $|\phi(1)\rangle$ respectively, where the superscript " $j$ " denotes the eight particles in the [8] irreducible representations.

The transformation between the two bases, the quark basis and the irreducible-representation particle basis (both are orthonormal), can be written in a $9 \times 9$ orthogonal matrix

$$
\left(\begin{array}{l}
\left|\phi^{\pi^{+}}\right\rangle \\
\left|\phi^{K^{+}}\right\rangle \\
\left|\phi^{\pi^{-}}\right\rangle \\
\left|\phi^{K^{0}}\right\rangle \\
\left|\phi^{K^{-}}\right\rangle \\
\left|\phi^{K^{0}}\right\rangle \\
\left|\phi^{\pi^{0}}\right\rangle \\
\left|\phi^{\eta_{8}}\right\rangle \\
\left|\phi^{\eta_{1}}\right\rangle
\end{array}\right)=\left(\begin{array}{ccccccccc}
1 & 0 & 0 & 0 & 0 & 0 & 0 & 0 & 0 \\
0 & 1 & 0 & 0 & 0 & 0 & 0 & 0 & 0 \\
0 & 0 & 1 & 0 & 0 & 0 & 0 & 0 & 0 \\
0 & 0 & 0 & 1 & 0 & 0 & 0 & 0 & 0 \\
0 & 0 & 0 & 0 & 1 & 0 & 0 & 0 & 0 \\
0 & 0 & 0 & 0 & 0 & 1 & 0 & 0 & 0 \\
0 & 0 & 0 & 0 & 0 & 0 & \frac{1}{\sqrt{2}} & \frac{-1}{\sqrt{2}} & 0 \\
0 & 0 & 0 & 0 & 0 & 0 & \frac{1}{\sqrt{6}} & \frac{1}{\sqrt{6}} & \frac{-2}{\sqrt{6}} \\
0 & 0 & 0 & 0 & 0 & 0 & \frac{1}{\sqrt{3}} & \frac{1}{\sqrt{3}} & \frac{1}{\sqrt{3}}
\end{array}\right)\left(\begin{array}{l}
|u \bar{d}\rangle \\
|u \bar{s}\rangle \\
|d \bar{u}\rangle \\
|d \bar{s}\rangle \\
|s \bar{u}\rangle \\
|s \bar{d}\rangle \\
|u \bar{u}\rangle \\
|d \bar{d}\rangle \\
|s \bar{s}\rangle
\end{array}\right) .
$$

The nine equations given by the matrix equation can also be written out as

$$
\left|M^{j}(8)\right\rangle=\sum_{\bar{q}, q^{\prime}}\left|\bar{q} q^{\prime}\right\rangle\left\langle\bar{q} q^{\prime} \mid M^{j}(8)\right\rangle
$$

and

$$
|M(1)\rangle=\sum_{\bar{q}, q^{\prime}}\left|\bar{q} q^{\prime}\right\rangle\left\langle\bar{q} q^{\prime} \mid M(1)\right\rangle
$$

where the summation is for $\bar{q}=\bar{u}, \bar{d}, \bar{s}$ and $q^{\prime}=u, d, s$. These equations are obtained simply by multiplying the left-hand side of (31) and (32) by

$$
\hat{I}=\sum_{\bar{q}, q^{\prime}}\left|\bar{q} q^{\prime}\right\rangle\left\langle\bar{q} q^{\prime}\right|,
$$


which is the completeness relation of the orthonormal $\left|q^{\prime} \bar{q}\right\rangle$-basis in the quark-antiquark vector space.

The irreducible-representation states in spin are related to the spin-product space by

$$
\left(\begin{array}{l}
\left|\chi^{+}(1)\right\rangle \\
\left|\chi^{-}(1)\right\rangle \\
\left|\chi^{0}(1)\right\rangle \\
|\chi(0)\rangle
\end{array}\right)=\left(\begin{array}{cccc}
1 & 0 & 0 & 0 \\
0 & 1 & 0 & 0 \\
0 & 0 & \frac{1}{\sqrt{2}} & \frac{1}{\sqrt{2}} \\
0 & 0 & \frac{1}{\sqrt{2}} & \frac{-1}{\sqrt{2}}
\end{array}\right)\left(\begin{array}{c}
\left|\frac{+1}{2} \frac{+1}{2}\right\rangle \\
\left|\frac{-1}{2} \frac{-1}{2}\right\rangle \\
\left|\frac{-1}{2} \frac{+1}{2}\right\rangle \\
\left|\frac{+1}{2} \frac{-1}{2}\right\rangle
\end{array}\right)
$$

and its inverse is trivially obtained (by using the transpose of the matrix)

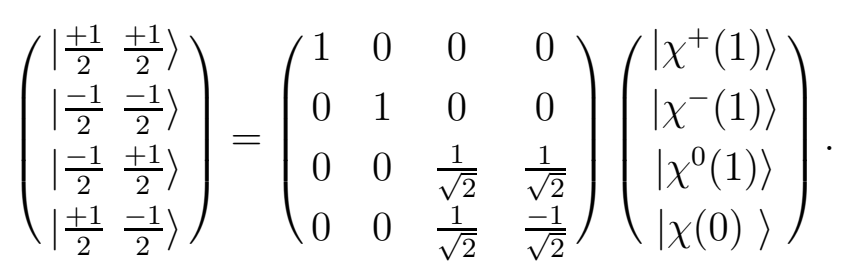

For pseudoscalar mesons, the wave functions are simply given by

$$
\begin{aligned}
|M(1)\rangle & =|\chi(0)\rangle|\phi(8)\rangle, \\
\left|M^{j}(8)\right\rangle & =|\chi(0)\rangle\left|\phi^{j}(8)\right\rangle,
\end{aligned}
$$

where the superscript $j$ indicates the eight different particles given in Eq. (30). 
III. Quark Diagram Scheme for $B_{c}(\overline{3}) \rightarrow B(10)+M(8)$

The light quarks of the charmed baryons belong to either a [3] or a [6] representation of the flavor $\mathrm{SU}(3)$. The $\Lambda_{c}^{+}, \Xi_{c}^{+A}$, and $\Xi_{c}^{0 A}$ form a $[\overline{3}]$ representation. They all decay weakly. The $\Omega_{c}^{0}, \Xi_{c}^{+S}, \Xi_{c}^{0 S}, \Sigma_{c}^{++}, \Sigma_{c}^{+}, \Sigma_{c}^{0}$ form a [6] representation; among them, however, only $\Omega_{c}^{0}$ decays weakly (the $\Sigma_{c}^{++,+, 0}$ decay strongly to the $\Lambda_{c}^{+}$of the $[\overline{3}]$ representation and the $\Xi_{c}^{+, 0}$ decay electromagnetically).

We shall first discuss the simpler case of the decuplet baryon being in the decay products. 


\section{III.a. Formalism}

Consider a particular charmed baryon $B_{c}^{m, i_{0}}$ decaying into an octet meson $M^{j_{0}}(8)$ and a decuplet baryon $B^{m, k_{0}}(10)$, where the subscript " 0 " signifies that we are discussing a specific baryon and a specific meson. The amplitude with the spin-projection $m, m^{\prime}$ summed over is

$$
\begin{aligned}
A\left(i_{0} \rightarrow j_{0} k_{0}\right) & \equiv \sum_{m, m^{\prime}}\left\langle B_{c}^{m, i_{0}}\left|\hat{H}_{W}\right| M^{j_{0}}\right\rangle\left|B^{m^{\prime}, k_{0}}(10)\right\rangle ; \text { using Eq. (25) for }\left|B^{m, k_{0}}(10)\right\rangle \\
& =\sum_{m, m^{\prime}}\left\langle B_{c}^{m, i_{0}}\left|\hat{H}_{W}\right| M^{j_{0}}(8)\right\rangle\left|\chi^{m^{\prime}}\left(\frac{3}{2}\right)_{S_{t}}\right\rangle\left|\psi^{k_{0}}(10)_{S_{t}}\right\rangle \text {; inserting Eq. (12), } \\
& =\sum_{m, m^{\prime}, q_{i}}\left\langle B_{c}^{m, i_{0}}\left|\hat{H}_{W}\right| M^{j_{0}}(8)\right\rangle\left|\chi^{m^{\prime}}\left(\frac{3}{2}\right)_{S_{t}}\right\rangle\left|q_{1} q_{2} q_{3}\right\rangle\left\langle q_{1} q_{2} q_{3} \mid \psi^{k_{0}}(10)_{S_{t}}\right\rangle ; \\
& \quad \sum_{m, m^{\prime}, q_{i}}\left\langle B_{c}^{m, i_{0}}\left|\hat{H}_{W}\right| \chi(0)\right\rangle\left|\phi^{j_{0}}(8)\right\rangle\left|\chi\left(\frac{3}{2}\right)_{S_{t}}\right\rangle\left|q_{1} q_{2} q_{3}\right\rangle\left\langle q_{1} q_{2} q_{3} \mid \psi^{k_{0}}(10)_{S_{t}}\right\rangle ; \\
& \quad \sum_{m, m^{\prime}, \bar{q}, q^{\prime}, q_{i}}\left\langle B_{c}^{m, i_{0}}\left|\hat{H}_{W}\right| \chi(0)\right\rangle\left|\chi^{m^{\prime}}\left(\frac{3}{2}\right)_{S_{t}}\right\rangle\left|\bar{q} q^{\prime}\right\rangle\left|q_{1} q_{2} q_{3}\right\rangle\left\langle\bar{q} q^{\prime} \mid \phi^{j_{0}}(8)\right\rangle \\
& \times\left\langle q_{1} q_{2} q_{3} \mid \psi^{k_{0}}(10)_{S_{t}}\right\rangle \\
& \equiv \sum_{\bar{q}, q^{\prime}, q_{i}} A\left(i_{0} \rightarrow \bar{q} q^{\prime} q_{1} q_{2} q_{3}\right)\left\langle\bar{q} q^{\prime} \mid \phi^{j_{0}}(8)\right\rangle\left\langle q_{1} q_{2} q_{3} \mid \psi^{k_{0}}(10)_{S_{t}}\right\rangle,
\end{aligned}
$$

where

$$
A\left(i_{0} \rightarrow \bar{q} q^{\prime} q_{1} q_{2} q_{3}\right) \equiv \sum_{m, m^{\prime}}\left\langle B_{c}^{m, i_{0}}\left|\hat{H}_{W}\right| \chi(0)\right\rangle\left|\chi^{m^{\prime}}\left(\frac{3}{2}\right)_{S_{t}}\right\rangle\left|\bar{q} q^{\prime} q_{1} q_{2} q_{3}\right\rangle
$$

are the quark-diagram amplitudes. Therefore, Eq. (37) gives the particle amplitudes $A\left(i_{0} \rightarrow\right.$ $\left.j_{0} k_{0}\right)$ of $B_{c}^{i_{0}}$ decaying into particles $M^{j_{0}}(8)$ and $B^{k_{0}}(10)$ in terms of the quark amplitudes $A\left(i_{0} \rightarrow \bar{q} q^{\prime} q_{1} q_{2} q_{3}\right)$ of $B_{c}^{i_{0}}$ decaying into quarks $\bar{q} q^{\prime} q_{1} q_{2} q_{3}$. The coefficients $\left\langle\bar{q} q^{\prime} \mid \phi^{j_{0}}(8)\right\rangle$ and $\left\langle q_{1} q_{2} q_{3} \mid \psi^{k_{0}}(10)_{S_{t}}\right\rangle$, each set of which forming elements of an orthogonal matrix, are those given in Eq. (30) and Eqs. (3) to (7), respectively.

Using the orthonormality of the coefficients, we can easily convert Eq. (37) to express the quark amplitudes in terms of the particle amplitudes

$$
A\left(i_{0} \rightarrow \bar{q} q^{\prime} q_{1} q_{2} q_{3}\right)=\sum_{j_{0}, k_{0}} A\left(i_{0} \rightarrow j_{0} k_{0}\right)\left\langle\phi^{j_{0}}(8) \mid \bar{q} q^{\prime}\right\rangle\left\langle q_{1} q_{2} q_{3} \mid \psi^{k_{0}}(10)_{S_{t}}\right\rangle
$$

using the orthonormality condition of the coefficients, which is the result of the orthonormality of the states.

We can also formulate the relation (37) in the basis given by Eqs. (13) and (14), which is also more convenient to apply since $\left|\psi^{k_{0}}(10)_{S_{t}}\right\rangle$ is totally symmetric. Replacing "inserting 
Eq. (12)" by "inserting Eq. (18)" in Eq. (37), we obtain

$$
A\left(i_{0} \rightarrow j_{0} k_{0}\right) \equiv \sum_{\bar{q}, q^{\prime}, q_{i}} A\left(i_{0} \rightarrow \bar{q} q^{\prime}\left\{q_{a} q_{b}\right\} q_{c}\right)\left\langle q^{\prime} \bar{q} \mid \phi^{j_{0}}(8)\right\rangle\left\langle\left\{q_{a} q_{b}\right\} q_{c} \mid \psi^{k_{0}}(10)_{S_{t}}\right\rangle
$$

where

$$
A\left(i_{0} \rightarrow \bar{q} q^{\prime},\left\{q_{a} q_{b}\right\} q_{c}\right) \equiv \sum_{m, m^{\prime}}\left\langle B_{c}^{m, i_{0}}\left|\hat{H}_{W}\right| \chi(0)\right\rangle\left|\chi^{m^{\prime}}\left(\frac{3}{2}\right)_{S_{t}}\right\rangle\left|\bar{q} q^{\prime},\left\{q_{a} q_{b}\right\} q_{c}\right\rangle
$$

Let us look more carefully at the amplitudes. For $q_{a}=q_{b}$,

$$
\begin{aligned}
A\left(i_{0} \rightarrow \bar{q} q^{\prime},\left\{q_{a} q_{b}\right\} q_{c}\right) & =A\left(i_{0} \rightarrow \bar{q} q^{\prime}, q_{a} q_{a} q_{c}\right) \\
& \equiv A_{S}\left(B_{c}(\overline{3}) \rightarrow B(10) M(8)\right)
\end{aligned}
$$

and for $q_{a} \neq q_{b}$,

$$
\begin{aligned}
A\left(i_{0} \rightarrow \bar{q} q^{\prime},\left\{q_{a} q_{b}\right\} q_{c}\right) & =\frac{1}{\sqrt{2}}\left[A\left(i_{0} \rightarrow \bar{q} q^{\prime}, q_{a} q_{b} q_{c}\right)+A\left(i_{0} \rightarrow \bar{q} q^{\prime}, q_{b} q_{a} q_{c}\right)\right] \\
& \equiv \sqrt{2} A_{S}\left(B_{c}(\overline{3}) \rightarrow B(10) M(8)\right),
\end{aligned}
$$

where we have used

$$
\begin{aligned}
A\left(i_{0} \rightarrow \bar{q} q^{\prime}, q_{a} q_{b} q_{c}\right) & =\frac{1}{2}\left[A\left(i_{0} \rightarrow \bar{q} q^{\prime}, q_{a} q_{b} q_{c}\right)+A\left(i_{0} \rightarrow \bar{q} q^{\prime}, q_{b} q_{a} q_{c}\right)\right]_{q_{a} \neq q_{b}} \\
& \equiv A_{S}\left(B_{c}(\overline{3}) \rightarrow B(10) M(8)\right)
\end{aligned}
$$

We shall see later that this assumption gives results consistent with those using the SU(3)-IR amplitudes. Eqs. (42) and (43) can be combined into one equation

$$
A\left(i_{0} \rightarrow \bar{q} q^{\prime},\left\{q_{a} q_{b}\right\} q_{c}\right)=\left[\sqrt{2}\left(1-\delta_{q_{a} q_{b}}\right)+\delta_{q_{a} q_{b}}\right] A_{S}\left(B_{c}(\overline{3}) \rightarrow B(10) M(8)\right)
$$

which we substitute into Eq. (40) and obtain

$$
\begin{aligned}
A\left(i_{0} \rightarrow j_{0} k_{0}\right) & \equiv \sum_{\bar{q}, q^{\prime}, q_{i}}\left[\sqrt{2}\left(1-\delta_{q_{a} q_{b}}\right)+\delta_{q_{a} q_{b}}\right] A_{S}\left(B_{c}(\overline{3}) \rightarrow B(10) M(8)\right) \\
& \times\left\langle q^{\prime} \bar{q} \mid \phi^{j_{0}}(8)\right\rangle\left\langle\left\{q_{a} q_{b}\right\} q_{c} \mid \psi^{k_{0}}(10)_{S_{t}}\right\rangle .
\end{aligned}
$$

Here in Eq. (37) and in Eq. (45) we see the important use of Eq. (12) and of Eq. (18) to convert particle-amplitudes to the quark-amplitudes.

One can easily show by diagram drawing that the $B_{c}(\overline{3}) \rightarrow B(10)+M(8)$ decays have contributions only from the $W$-exchange and the horizontal $W$-loop diagrams, i.e., the $\mathcal{C}$ and $\mathcal{E}$ types of amplitudes. In the $\mathcal{A}$ and $\mathcal{B}$ amplitudes, the two spectator quarks that are 
antisymmetrized in the initial charmed baryon state remain to be antisymmetrized after the weak-interaction decay and cannot contribute to make an $B(10)$ whose wave function is totally symmetric. In the $\mathcal{C}$ and $\mathcal{E}$ types of amplitudes, an appropriate quark pair $\bar{q}_{0} q_{0}$ is created so that the $\bar{q}_{0}$ will combine with one of the quarks originated from the initial quark to form the meson $j_{0}$. Depending upon where the pair $\bar{q}_{0} q_{0}$ can be inserted in the diagrams, we have different types of $\mathcal{C}_{S}$ and $\mathcal{E}_{S}$ of amplitudes; $\mathcal{C}_{1 S}$ for $\bar{q}_{0}$ forming a meson with a spectator quark (which does not contribute in this case of $B(10)$ in the final state); $\mathcal{C}_{2 S}$ for $\bar{q}_{0}$ forming a meson with the weak-interacting non-charmed quark; $\mathcal{C}_{S}^{\prime}$ for $\bar{q}_{0}$ forming a meson with the quark decayed from the charmed quark; $\mathcal{E}_{S}$ for $\bar{q}_{0}$ forming a meson with the a spectator quark; and $\mathcal{E}_{S}^{\prime}$ for $\bar{q}_{0}$ forming a meson with the quark decayed from the charmed quark. The quark $q_{0}$ from the pair creation will form with the other two quarks to become the final baryon $k_{0}$. Thus in Eq. (45) only $q_{1}$ and $q_{2}$ are summed over and Eq. (45) becomes

$$
\begin{aligned}
A\left(i_{0}\right. & \left.\rightarrow j_{0} k_{0}\right) \\
& =\mathcal{C}_{2 S}\left(B_{c}(\overline{3}) \rightarrow B(10) M(8)\right)\left[\sqrt{2}\left(1-\delta_{q_{1} q_{3}}\right)+\delta_{q_{1} q_{3}}\right]\left\langle\bar{q}_{0} q_{2}^{\prime} \mid \phi^{j_{0}}(8)\right\rangle\left\langle\left\{q_{1} q_{3}\right\} q_{0} \mid \psi^{k_{0}}(10)_{S_{t}}\right\rangle \\
& +\mathcal{C}_{S}^{\prime}\left(B_{c}(\overline{3}) \rightarrow B(10) M(8)\right)\left[\sqrt{2}\left(1-\delta_{q_{1} q_{2}^{\prime}}\right)+\delta_{q_{1} q_{2}^{\prime}}\right]\left\langle\bar{q}_{0} q_{3} \mid \phi^{j_{0}}(8)\right\rangle\left\langle\left\{q_{1} q_{2}^{\prime}\right\} q_{0} \mid \psi^{k_{0}}(10)_{S_{t}}\right\rangle \\
& +\mathcal{E}_{S}\left(B_{c}(\overline{3}) \rightarrow B(10) M(8)\right)\left[\sqrt{2}\left(1-\delta_{q_{3} q_{1}}\right)+\delta_{q_{3} q_{1}}\right]\left\langle\bar{q}_{0} q_{2} \mid \phi^{j_{0}}(8)\right\rangle\left\langle\left\{q_{3} q_{1}\right\} q_{0} \mid \psi^{k_{0}}(10)_{S_{t}}\right\rangle .
\end{aligned}
$$

Using (46) for $B_{c}(\overline{3}) \rightarrow B(10)+M(8)$ decays, we obtain column two of Tables 1a, $1 \mathrm{~b}$ and 1c. (In these tables we have dropped the parenthesis that specify the decay of $B_{c}(\overline{3}) \rightarrow B(10) M(8)$.) We see that all $B_{c}(\overline{3}) \rightarrow B(10) M(8)$ decays, fifty-five of them, can be expressed in terms of the three unknown amplitudes: $\mathcal{C}_{2 S}, \mathcal{C}_{S}^{\prime}$ and $\mathcal{E}_{S}$. Therefore, we obtain many relations among the particle decay amplitudes as shown in the next section.

Next we make an attempt to include and parametrize the effects of final-state interactions and $\mathrm{SU}(3)$ symmetry breaking. For final-state interactions we introduce an explicit factor $e^{i \delta}$ to each isospin partial-wave amplitude. These phase shifts $\delta$ 's in general have both real and imaginary parts; the imaginary component indicates the inelastic effect. SU(3)-violation effects can manifest in several places. For example, the quark diagrams with $s \bar{s}$ insertion are a priori different from those arising from $u \bar{u}$ or $d \bar{d}$ insertion. However, for the simplicity of the presentation of the Tables in the present paper, we parametrize $\mathrm{SU}(3)$ symmetry breaking only in the $\mathcal{E}$ type of quark diagrams whose presence (i.e. contributions to the quark-mixing-singly-suppressed decay modes) are solely due to $\mathrm{SU}(3)$ violation effects.

\section{III.b. Results and Tables}

In the absence of effects from $\mathrm{SU}(3)$ breaking and final-state interactions, the following 
relations can be obtained from the second column of Tables 1a to 1c, namely

$$
\begin{gathered}
\left|A\left(\Lambda_{c}^{+} \rightarrow \Sigma^{*+} \eta_{8}\right)\right|^{2}=\left|A\left(\Xi^{0 A} \rightarrow \Xi^{* 0} \eta_{8}\right)\right|^{2} \\
\left.\left|A\left(\Xi_{c}^{0 A} \rightarrow \Omega^{-} K^{+}\right)\right|^{2}=3\left|A\left(\Xi_{c}^{0 A} \rightarrow \Xi^{*-} \pi^{+}\right)\right|^{2}=3 \mid \Lambda_{c}^{+} \rightarrow \Xi^{* 0} K^{+}\right)\left.\right|^{2} \\
=6\left|A\left(\Xi_{c}^{0 A} \rightarrow \Xi^{* 0} \pi^{0}\right)\right|^{2}=6\left|A\left(\Lambda_{c}^{+} \rightarrow \Sigma^{*+} \pi^{0}\right)\right|^{2}=6\left|A\left(\Lambda_{c}^{+} \rightarrow \Sigma^{* 0} \pi^{+}\right)\right|^{2} \\
\left|A\left(\Lambda_{c}^{+} \rightarrow \Delta^{++} K^{-}\right)\right|^{2}=3\left|A\left(\Lambda_{c}^{+} \rightarrow \Delta^{+} \bar{K}^{0}\right)\right|^{2} \\
=3\left|A\left(\Xi_{c}^{0 A} \rightarrow \Sigma^{*+} K^{-}\right)\right|^{2}=6\left|A\left(\Xi_{c}^{0 A} \rightarrow \Sigma^{* 0} \bar{K}^{0}\right)\right|^{2}
\end{gathered}
$$

for quark-mixing-allowed modes;

$$
\begin{gathered}
\left|A\left(\Xi_{c}^{0 A} \rightarrow \Sigma^{* 0} \pi^{0}\right)\right|^{2}=3\left|A\left(\Xi_{c}^{0 A} \rightarrow \Sigma^{* 0} \eta_{8}\right)\right|^{2}, \\
\left|A\left(\Xi_{c}^{0 A} \rightarrow \Sigma^{*-} \pi^{+}\right)\right|^{2}=\left|A\left(\Xi_{c}^{0 A} \rightarrow \Xi^{*-} K^{+}\right)\right|^{2}=4\left|A\left(\Lambda_{c}^{+} \rightarrow \Delta^{0} \pi^{+}\right)\right|^{2} \\
=4\left|A\left(\Xi_{c}^{+A} \rightarrow \Xi^{* 0} K^{+}\right)\right|^{2}=8\left|A\left(\Lambda_{c}^{+} \rightarrow \Sigma^{* 0} K^{+}\right)\right|^{2} \\
=8\left|A\left(\Xi_{c}^{+A} \rightarrow \Sigma^{* 0} \pi^{+}\right)\right|^{2}=8\left|A\left(\Xi_{c}^{+A} \rightarrow \Sigma^{*+} \pi^{0}\right)\right|^{2}, \\
=3\left|A\left(\Lambda_{c}^{+} \rightarrow \Delta^{++} \pi^{-}\right)\right|^{2}=\left|A\left(\Xi_{c}^{+A} \rightarrow \Delta^{+} \bar{K}^{0}\right)\right|^{2}=3\left|A\left(\Xi_{c}^{0 A} \rightarrow \Delta^{0} \bar{K}^{0}\right)\right|^{2}=3\left|A\left(\Xi_{c}^{+} \rightarrow \Sigma_{c}^{*+} \rightarrow \Xi^{* 0} K^{0}\right)\right|^{2} \\
=3\left|A\left(\Xi_{c}^{0 A} \rightarrow \Sigma^{*+} \pi^{-}\right)\right|^{2}=3\left|A\left(\Xi_{c}^{0 A} \rightarrow \Delta^{+} K^{-}\right)\right|^{2}
\end{gathered}
$$

for quark-mixing-suppressed modes;

$$
\begin{aligned}
\left|A\left(\Xi_{c}^{+A} \rightarrow \Delta^{+} \eta_{8}\right)\right|^{2} & =\left|A\left(\Xi_{c}^{0 A} \rightarrow \Delta^{0} \eta_{8}\right)\right|^{2}, \\
\left|A\left(\Xi_{c}^{0 A} \rightarrow \Delta^{0} \pi^{0}\right)\right|^{2} & =2\left|A\left(\Xi_{c}^{+A} \rightarrow \Delta^{+} \pi^{0}\right)\right|^{2}, \\
\left|A\left(\Xi_{c}^{+A} \rightarrow \Delta^{++} \pi^{-}\right)\right|^{2} & =3\left|A\left(\Xi_{c}^{+A} \rightarrow \Sigma^{*+} K^{0}\right)\right|^{2} \\
=3\left|A\left(\Xi_{c}^{0 A} \rightarrow \Delta^{+} \pi^{-}\right)\right|^{2} & =6\left|A\left(\Xi_{c}^{0 A} \rightarrow \Sigma^{* 0} K^{0}\right)\right|^{2}, \\
\left|A\left(\Xi_{c}^{0 A} \rightarrow \Delta^{-} \pi^{+}\right)\right|^{2} & =3\left|A\left(\Xi_{c}^{+A} \rightarrow \Delta^{0} \pi^{+}\right)\right|^{2} \\
=3\left|A\left(\Xi_{c}^{0 A} \rightarrow \Sigma^{*-} K^{+}\right)\right|^{2} & =6\left|A\left(\Xi_{c}^{+A} \rightarrow \Sigma^{* 0} K^{+}\right)\right|^{2}
\end{aligned}
$$

for quark-mixing-doubly-suppressed modes, and many relations between quark-mixing-allowed, -suppressed, and -doubly-suppressed decay modes, for example

$$
\begin{aligned}
\left|A\left(\Lambda_{c}^{+} \rightarrow \Delta^{0} \pi^{+}\right)\right|^{2} & =2 s_{1}^{2}\left|A\left(\Lambda_{c}^{+} \rightarrow \Sigma^{*+} \pi^{0}\right)\right|^{2}, \\
\left|A\left(\Lambda_{c}^{+} \rightarrow \Delta^{++} \pi^{-}\right)\right|^{2} & =s_{1}^{2}\left|A\left(\Lambda_{c}^{+} \rightarrow \Delta^{++} K^{-}\right)\right|^{2}, \\
\left|A\left(\Xi_{c}^{+A} \rightarrow \Sigma^{* 0} K^{+}\right)\right|^{2} & =s_{1}^{4}\left|A\left(\Lambda_{c}^{+} \rightarrow \Sigma^{*+} \pi^{0}\right)\right|^{2}, \\
\left|A\left(\Xi_{c}^{+A} \rightarrow \Delta^{++} \pi^{-}\right)\right|^{2} & =s_{1}^{4}\left|A\left(\Lambda_{c}^{+} \rightarrow \Delta^{++} K^{-}\right)\right|^{2} .
\end{aligned}
$$


Several comments are in order. (i) Table 1.a and relations (47) for quark-mixing-allowed decays $B_{c}(\overline{3}) \rightarrow B(10)+M(8)$ are also obtained previously by Kohara [10] and are in agree with ours. (ii) Though all the above quark-diagram relations are obtained in the absence of effects from $\mathrm{SU}(3)$ violations and final-state interactions, however, if each quarkdiagram amplitude in the $\mathrm{SU}(3)$ relation contains only a single isospin phase shift, then such a relation holds even in the presence of final-state interactions because of SU(3) symmetry for phase shifts. Examples are the first relation in Eqs. (47) and (49). The same is true for the relations obtained below. (iii) We note that the quark-mixing-allowed decays of an antitriplet charmed baryon into a decuplet baryon and a pseudoscalar meson can occur only through $W$-exchange diagrams. The experimental measurement of $\Lambda_{c}^{+} \rightarrow \Delta^{++} K^{-}[2]$ indicates that the $W$-exchange mechanism plays a significant role in charmed baryon decays. (iv) The quark-mixing-allowed decays of $\Xi_{c}^{+A}$ and quark-mixing-doubly-suppressed decays of $\Lambda_{c}^{+}$into a decuplet baryon are prohibited in the quark-diagram scheme:

$$
\begin{aligned}
\left|A\left(\Xi_{c}^{+A} \rightarrow \Sigma^{*+} \bar{K}^{0}\right)\right|^{2} & =0, \quad\left|A\left(\Xi_{c}^{+A} \rightarrow \Xi^{* 0} \pi^{+}\right)\right|^{2}=0, \\
\left|A\left(\Lambda_{c}^{+} \rightarrow \Delta^{+} K^{0}\right)\right|^{2} & =0, \quad\left|A\left(\Lambda_{c}^{+} \rightarrow \Delta^{0} K^{+}\right)\right|^{2}=0 .
\end{aligned}
$$

In the SU(3)-IR approach of Savage and Springer (SS) [6], these decays are governed by the reduced matrix element $\alpha$ defined in Eq. (17) of Ref. [6]. However, we see that they are forbidden in the quark-diagram scheme since they are given by the quark diagram $\mathcal{A}$ or $\mathcal{B}^{\prime}$ and they give zero contribution, as we discussed before, because of the un-matching symmetry properties of the antitriplet charmed baryon and the decuplet baryon. Furthermore, we note that the SU(3)-IR approach of SS will predict the above relations (48-51) only if the reduced matrix elements $\alpha$ and $\gamma$ make no contributions. As a consequence, there are only two independent $\mathrm{SU}(3)$ reduced matrix elements $\beta$ and $\delta$. The quark-diagram amplitudes and the $\mathrm{SU}(3)$-symmetry parameters are related by

$$
\beta=\frac{1}{2}\left(\mathcal{C}_{S}^{\prime}+\mathcal{C}_{2 S}\right), \quad \delta=\frac{1}{2}\left(\mathcal{C}_{S}^{\prime}-\mathcal{C}_{2 S}\right), \quad \alpha=\gamma=0 .
$$


IV. Quark Diagram Scheme for $B_{c}(\overline{3}) \rightarrow B(8)+M(8)$

\section{IV.a. The Formalism}

The formalism is very similar to that given in Sect. III.a. for the decuplet baryon in the final state except for the complication that the octet baryons are made up with two orthonormal parts, Eq. (26). We shall see that all it does is that each type of the quark amplitude $A$ will be made up of two independent ones, the symmetric and the antisymmetric. Following the similar procedure used in Eqs. (37) and (50), we derive

$$
\begin{aligned}
& A\left(i_{0} \rightarrow j_{0} k_{0}\right)=\sum_{m, m^{\prime}}\left\langle B_{c}^{m, i_{0}}\left|\hat{H}_{W}\right| M^{j_{0}}(8)\right\rangle\left|B^{m^{\prime}, k_{0}}(8)\right\rangle \\
& =\sum_{m, m^{\prime}}\left\langle B_{c}^{m, i_{0}}\left|\hat{H}_{W}\right| M^{j_{0}}(8)\right\rangle\left(a\left|\chi^{m^{\prime}}\left(\frac{1}{2}\right)_{A}\right\rangle\left|\psi^{k_{0}}(8)_{A}\right\rangle+b\left|\chi^{m^{\prime}}\left(\frac{1}{2}\right)_{S}\right\rangle\left|\psi^{k_{0}}(8)_{S}\right\rangle\right) \\
& =\sum_{m, m^{\prime}, q_{i}} a\left\langle B_{c}^{m, i_{0}}\left|H_{W}\right| M^{j_{0}}(8)\right\rangle\left|\chi^{m^{\prime}}\left(\frac{1}{2}\right)_{A}\right\rangle\left|q_{1} q_{2} q_{3}\right\rangle\left\langle q_{1} q_{2} q_{3} \mid \psi^{k_{0}}(8)_{A}\right\rangle \\
& +\sum_{m, m^{\prime}, q_{i}} b\left\langle B_{c}^{m, i_{0}}\left|H_{W}\right| M^{j_{0}}(8)\right\rangle\left|\chi^{m^{\prime}}\left(\frac{1}{2}\right)_{S}\right\rangle\left|q_{1} q_{2} q_{3}\right\rangle\left\langle q_{1} q_{2} q_{3} \mid \psi^{k_{0}}(8)_{S}\right\rangle \\
& =\sum_{m, m^{\prime}, q_{i}} a\left\langle B_{c}^{m, i_{0}}\left|H_{W}\right| M^{j_{0}}(8)\right\rangle\left|\chi^{m^{\prime}}\left(\frac{1}{2}\right)_{A}\right\rangle\left|\left[q_{1} q_{2}\right] q_{3}\right\rangle\left\langle\left[q_{1} q_{2}\right] q_{3} \mid \psi^{k_{0}}(8)_{A}\right\rangle \\
& +\sum_{m, m^{\prime}, q_{i}} b\left\langle B_{c}^{m, i_{0}}\left|H_{W}\right| M^{j_{0}}(8)\right\rangle\left|\chi^{m^{\prime}}\left(\frac{1}{2}\right)_{S}\right\rangle\left|\left\{q_{1} q_{2}\right\} q_{3}\right\rangle\left\langle\left\{q_{1} q_{2}\right\} q_{3} \mid \psi^{k_{0}}(8)_{S}\right\rangle .
\end{aligned}
$$

To decompose the meson state into the $q^{\prime} \bar{q}$ state, we insert in Eq. (53) the completeness relation Eq. (33) and obtain

$$
\begin{aligned}
& A\left(i_{0} \rightarrow j_{0} k_{0}\right)=\sum_{m, m^{\prime}, \bar{q}, q^{\prime}, q_{i}} b^{*}\left\langle B_{c}^{m, i_{0}}\left|\hat{H}_{W}\right| \chi\left(0^{-}\right)\right\rangle\left|\bar{q} q^{\prime}\right\rangle\left|\chi^{m^{\prime}}\left(\frac{1}{2}\right)_{A}\right\rangle\left|\left[q_{1} q_{2}\right] q_{3}\right\rangle \\
& \times\left\langle\bar{q} q^{\prime} \mid \phi^{j_{0}}(8)\right\rangle\left\langle\left[q_{1} q_{2}\right] q_{3} \mid \psi^{k_{0}}(8)_{A}\right\rangle \\
& -\sum_{m, m^{\prime}, \bar{q}, q^{\prime}, q_{i}} a^{*}\left\langle B_{c}^{m, i_{0}}\left|\hat{H}_{W}\right| \chi\left(0^{-}\right)\right\rangle\left|\bar{q} q^{\prime}\right\rangle\left|\chi^{m^{\prime}}\left(\frac{1}{2}\right)_{S}\right\rangle\left|\left\{q_{1} q_{2}\right\} q_{3}\right\rangle \\
& \times\left\langle\bar{q} q^{\prime} \mid \phi^{j_{0}}(8)\right\rangle\left\langle q_{1} q_{2} q_{3} \mid \psi^{k_{0}}(8)_{S}\right\rangle \\
& \equiv \sum_{\bar{q}, q^{\prime}, q_{i}} A\left(i_{0} \rightarrow \bar{q} q^{\prime}\left[\begin{array}{ll}
q_{1} & q_{2}
\end{array}\right] q_{3}\right)\left\langle\bar{q} q^{\prime} \mid \phi^{j_{0}}(8)\right\rangle\left\langle\left[\begin{array}{ll}
q_{1} & q_{2}
\end{array}\right] q_{3} \mid \psi^{k_{0}}(8)_{A}\right\rangle \\
& +\sum_{\bar{q}, q^{\prime}, q_{i}} A\left(i_{0} \rightarrow \bar{q} q^{\prime}\left\{\begin{array}{ll}
q_{1} & q_{2}
\end{array}\right\} q_{3}\right)\left\langle\bar{q} q^{\prime} \mid \phi^{j_{0}}(8)\right\rangle\left\langle\left\{\begin{array}{ll}
q_{1} & q_{2}
\end{array}\right\} q_{3} \mid \psi^{k_{0}}(8)_{S}\right\rangle,
\end{aligned}
$$

where

$$
\begin{aligned}
A\left(i_{0} \rightarrow \bar{q} q^{\prime}\left[\begin{array}{ll}
q_{1} & q_{2}
\end{array}\right] q_{3}\right) & \equiv \sum_{m, m^{\prime}} b^{*}\left\langle B_{c}^{m, i_{0}}\left|\hat{H}_{W}\right| \chi\left(0^{-}\right)\right\rangle\left|\bar{q} q^{\prime}\right\rangle\left|\chi^{m^{\prime}}\left(\frac{1}{2}\right)_{A}\right\rangle\left|\left[q_{1} q_{2}\right] q_{3}\right\rangle \\
& =A_{A}\left(B_{c}(\overline{3}) \rightarrow B(8) M(8)\right),
\end{aligned}
$$


and

$$
\begin{aligned}
A\left(i_{0} \rightarrow \bar{q} q^{\prime}\left\{q_{1} q_{2}\right\} q_{3}\right) & \equiv \sum_{m, m^{\prime}} b^{*}\left\langle B_{c}^{m, i_{0}}\left|\hat{H}_{W}\right| \chi\left(0^{-}\right)\right\rangle\left|\bar{q} q^{\prime}\right\rangle\left|\chi^{m^{\prime}}\left(\frac{1}{2}\right)_{A}\right\rangle\left|\left\{q_{1} q_{2}\right\} q_{3}\right\rangle \\
& =\left[\sqrt{2}\left(1-\delta_{q_{1} q_{2}}\right)+\delta_{q_{1} q_{2}}\right] A_{S}\left(B_{c}(\overline{3}) \rightarrow B(8) M(8)\right) .
\end{aligned}
$$

Now the decay amplitudes into particles are related to decay amplitudes into quarks.

Therefore, the important result we have established is that for the decays into the $B(8)$, the quark diagrams have two independent types: the symmetric and the antisymmetric, $A_{A}$ and $A_{S}$. This result is independent of what particles the $B(8)$ 's decay from or are associated with.

Let us discuss now specifically what types of quark diagram amplitudes will contribute. For $B_{c}(\overline{3}) \rightarrow B(8)+M(8)$, the two initial non-charmed quarks, say $q_{1}$ and $q_{2}$, are antisymmetric in flavor. In diagram $\mathcal{A}, q_{1}$ and $q_{2}$ are spectators; therefore, they stay antisymmetric in the final state. We denote the quark arising from the charmed quark decay as $q_{3}$, and the quark-antiquark pair from the $W$ as $\bar{q}_{0} q_{0}^{\prime}$. In diagram $\mathcal{B}^{\prime}$ (the superscript "'” signifies that the quark $q_{3}$ coming from the charmed quark decay contributes to the final meson formation rather than the final baryon formation), $q_{1}$ and $q_{2}$ are also spectators; therefore, they stay antisymmetric in the final product. In diagram $\mathcal{B}, q_{3}$ and $q_{0}^{\prime}$ are forced to be flavor antisymmetric due to the Pati-Woo theorem [11], so are the quark pair $q_{1}^{\prime} q_{3}$ in $\operatorname{diagram} \mathcal{C}_{1}$. Note that the quark-diagram amplitudes $\mathcal{B}_{S}^{\prime}$ and $\mathcal{C}_{1 S}$ vanish because of the Pati-Woo theorem which results from the fact that the $(V-A) \times(V-A)$ structure of weak interactions is invariant under the Fierz transformation and that the baryon wave function is color antisymmetric. This theorem requires that the quark pair in a baryon produced by weak interactions be antisymmetric in flavor. Putting together all these information and referring to Fig. 2, we find the detailed expression for Eq. (54)

$$
\begin{aligned}
A\left(i_{0} \rightarrow j_{0} k_{0}\right) & =\mathcal{A}_{A}\left(B_{c}(\overline{3}) \rightarrow B(8) M(8)\right)\left\langle\bar{q}_{0} q_{0}^{\prime} \mid \phi^{j_{0}}(8)\right\rangle\left\langle\left[q_{1} q_{2}\right] q_{3} \mid \psi^{k_{0}}(8)_{A}\right\rangle \\
& +\mathcal{B}_{A}^{\prime}\left(B_{c}(\overline{3}) \rightarrow B(8) M(8)\right)\left\langle\bar{q}_{0} q_{3}^{\prime} \mid \phi^{j_{0}}(8)\right\rangle\left\langle\left[q_{1} q_{2}\right] q_{0}^{\prime} \mid \psi^{k_{0}}(8)_{A}\right\rangle \\
& +\mathcal{B}_{A}\left(B_{c}(\overline{3}) \rightarrow B(8) M(8)\right)\left\langle\bar{q}_{0} q_{2} \mid \phi^{j_{0}}(8)\right\rangle\left\langle\left[q_{1} q_{3}\right] q_{0}^{\prime} \mid \psi^{k_{0}}(8)_{A}\right\rangle \\
& +\mathcal{C}_{1 A}\left(B_{c}(\overline{3}) \rightarrow B(8) M(8)\right)\left\langle\bar{q}_{0} q_{2} \mid \phi^{j_{0}}(8)\right\rangle\left\langle\left[q_{1}^{\prime} q_{3}\right] q_{0} \mid \psi^{k_{0}}(8)_{A}\right\rangle \\
& +\mathcal{C}_{2 A}\left(B_{c}(\overline{3}) \rightarrow B(8) M(8)\right)\left\langle\bar{q}_{0} q_{2}^{\prime} \mid \phi^{j_{0}}(8)\right\rangle\left\langle\left[q_{1} q_{3}\right] q_{0} \mid \psi^{k_{0}}(8)_{A}\right\rangle \\
& +\mathcal{C}_{2 S}\left(B_{c}(\overline{3}) \rightarrow B(8) M(8)\right)\left\langle\bar{q}_{0} q_{2}^{\prime} \mid \phi^{j_{0}}(8)\right\rangle\left\langle\left\{q_{1} q_{3}\right\} q_{0} \mid \psi^{k_{0}}(8)_{S}\right\rangle\left[\sqrt{2}\left(1-\delta_{q_{1} q_{3}}\right)+\delta_{q_{1} q_{3}}\right] \\
& +\mathcal{C}_{A}^{\prime}\left(B_{c}(\overline{3}) \rightarrow B(8) M(8)\right)\left\langle\bar{q}_{0} q_{3} \mid \phi^{j_{0}}(8)\right\rangle\left\langle\left\{q_{1} q_{2}^{\prime}\right\} q_{3} \mid \psi^{k_{0}}(8)_{A}\right\rangle \\
& +\mathcal{C}_{S}^{\prime}\left(B_{c}(\overline{3}) \rightarrow B(8) M(8)\right)\left\langle\bar{q}_{0} q_{3} \mid \phi^{j_{0}}(8)\right\rangle\left\langle\left\{q_{1} q_{2}^{\prime}\right\} q_{0} \mid \psi^{k_{0}}(8)_{S}\right\rangle\left[\sqrt{2}\left(1-\delta_{q_{1} q_{2}^{\prime}}\right)+\delta_{q_{1} q_{2}^{\prime}}\right] \\
& +\mathcal{E}_{A}\left(B_{c}(\overline{3}) \rightarrow B(8) M(8)\right)\left\langle\bar{q}_{0} q_{2} \mid \phi^{j_{0}}(8)\right\rangle\left\langle\left[q_{3} q_{1}\right] q_{0} \mid \psi^{k_{0}}(8)_{A}\right\rangle
\end{aligned}
$$




$$
\begin{aligned}
& +\mathcal{E}_{S}\left(B_{c}(\overline{3}) \rightarrow B(8) M(8)\right)\left\langle\bar{q}_{0} q_{2} \mid \phi^{j_{0}}(8)\right\rangle\left\langle\left\{q_{3} q_{1}\right\} q_{0} \mid \psi^{k_{0}}(8)_{S}\right\rangle\left[\sqrt{2}\left(1-\delta_{q_{3} q_{1}}\right)+\delta_{q_{3} q_{1}}\right] \\
& +\mathcal{E}_{A}^{\prime}\left(B_{c}(\overline{3}) \rightarrow B(8) M(8)\right)\left\langle\bar{q}_{0} q_{3} \mid \phi^{j_{0}}(8)\right\rangle\left\langle\left[q_{1} q_{2}\right] q_{0} \mid \psi^{k_{0}}(8)_{A}\right\rangle .
\end{aligned}
$$

Applying this to all the $B_{c}(\overline{3}) \rightarrow B(8) M(8)$ decays, we can express all the 58 decays in terms of the eleven unknown amplitudes in (57) (see also Table 2).

\section{IV.b. Results and Tables}

From the second column of Tabs. 2a-2c, which gives the results in the absence of effects from $\mathrm{SU}(3)$ violations and final state interactions, we have the following relations :

$$
\begin{aligned}
\left|A\left(\Xi_{c}^{0 A} \rightarrow \Sigma^{-} \pi^{+}\right)\right|^{2} & =\left|A\left(\Xi_{c}^{0 A} \rightarrow \Xi^{-} K^{+}\right)\right|^{2}, \\
\left|A\left(\Xi_{c}^{0 A} \rightarrow n \bar{K}^{0}\right)\right|^{2} & =\left|A\left(\Xi_{c}^{0 A} \rightarrow \Xi^{0} K^{0}\right)\right|^{2}, \\
\left|A\left(\Xi_{c}^{0 A} \rightarrow \Sigma^{+} \pi^{-}\right)\right|^{2} & =\left|A\left(\Xi_{c}^{0 A} \rightarrow p K^{-}\right)\right|^{2}, \\
\left|A\left(\Xi_{c}^{+A} \rightarrow p \bar{K}^{0}\right)\right|^{2} & =\left|A\left(\Lambda_{c}^{+} \rightarrow \Sigma^{+} K^{0}\right)\right|^{2}, \\
\left|A\left(\Xi_{c}^{+A} \rightarrow \Xi^{0} K^{+}\right)\right|^{2} & =\left|A\left(\Lambda_{c}^{+} \rightarrow n \pi^{+}\right)\right|^{2}, \\
\left|A\left(\Xi_{c}^{0 A} \rightarrow \Lambda^{0} \eta_{8}\right)\right|^{2} & =\left|A\left(\Xi_{c}^{0 A} \rightarrow \Sigma^{0} \pi^{0}\right)\right|^{2},
\end{aligned}
$$

for quark-mixing-suppressed modes,

$$
\begin{aligned}
& \left|A\left(\Xi_{c}^{+A} \rightarrow \Sigma^{+} K^{0}\right)\right|^{2}=2\left|A\left(\Xi_{c}^{0 A} \rightarrow \Sigma^{0} K^{0}\right)\right|^{2}, \\
& \left|A\left(\Xi_{c}^{0 A} \rightarrow \Sigma^{-} K^{+}\right)\right|^{2}=2\left|A\left(\Xi_{c}^{+A} \rightarrow \Sigma^{0} K^{+}\right)\right|^{2},
\end{aligned}
$$

for quark-mixing-doubly-suppressed modes, and relations between the squares of quarkmixing-allowed, -suppressed, and quark-mixing-doubly-suppressed amplitudes:

$$
\begin{aligned}
\left|A\left(\Lambda_{c}^{+} \rightarrow p \eta_{0}\right)\right|^{2} & =s_{1}^{2}\left|A\left(\Lambda_{c}^{+} \rightarrow \Sigma^{+} \eta_{0}\right)\right|^{2}, \\
\left|A\left(\Xi_{c}^{0 A} \rightarrow \Xi^{-} K^{+}\right)\right|^{2} & =s_{1}^{2}\left|A\left(\Xi_{c}^{0 A} \rightarrow \Xi^{-} \pi^{+}\right)\right|^{2}, \\
\left|A\left(\Xi_{c}^{0 A} \rightarrow \Sigma^{+} \pi^{-}\right)\right|^{2} & =s_{1}^{2}\left|A\left(\Xi_{c}^{0 A} \rightarrow \Sigma^{+} K^{-}\right)\right|^{2}, \\
\left|A\left(\Xi_{c}^{+A} \rightarrow \Sigma^{+} K^{0}\right)\right|^{2} & =s_{1}^{4}\left|A\left(\Lambda_{c}^{+} \rightarrow p \bar{K}^{0}\right)\right|^{2}, \\
\left|A\left(\Xi_{c}^{+A} \rightarrow n \pi^{+}\right)\right|^{2} & =s_{1}^{4}\left|A\left(\Lambda_{c}^{+} \rightarrow \Xi^{0} K^{+}\right)\right|^{2}, \\
\left|A\left(\Xi_{c}^{0 A} \rightarrow \Sigma^{-} K^{+}\right)\right|^{2} & =s_{1}^{4}\left|A\left(\Xi_{c}^{0 A} \rightarrow \Xi^{-} \pi^{+}\right)\right|^{2}, \\
\left|A\left(\Xi_{c}^{0 A} \rightarrow p \pi^{-}\right)\right|^{2} & =s_{1}^{4}\left|A\left(\Xi_{c}^{0 A} \rightarrow \Sigma^{+} K^{-}\right)\right|^{2}, \\
\left|A\left(\Lambda_{c}^{+} \rightarrow n K^{+}\right)\right|^{2} & =s_{1}^{4}\left|A\left(\Xi_{c}^{+A} \rightarrow \Xi^{0} \pi^{+}\right)\right|^{2}, \\
\left|A\left(\Lambda_{c}^{+} \rightarrow p K^{0}\right)\right|^{2} & =s_{1}^{4}\left|A\left(\Xi_{c}^{+A} \rightarrow \Sigma^{+} \bar{K}^{0}\right)\right|^{2}, \\
\left|A\left(\Xi_{c}^{+} \rightarrow p \eta_{0}\right)\right|^{2} & =s_{1}^{4}\left|A\left(\Lambda_{c}^{+} \rightarrow \Sigma^{+} \eta_{0}\right)\right|^{2},
\end{aligned}
$$

where $s_{1}=\sin \theta_{1}$, and $\theta_{1}$ is the usual quark-mixing angle. 
Note that the above quark-diagram relations can also be reproduced in the SU(3) Hamiltonian approach of Savage and Springer (SS) [6] Đ except for Eq. (59) and the first and last relations in Eq. (60). We believe that when the use of the SU(3) Hamiltonian in which the symmetry amplitudes are tensor decomposed is done correctly to incorporate the symmetry properties of the baryon wave function, the reduced matrix element $a$ defined in Ref.[6] should not contribute and all aforementioned SU(3) quark-diagram results will be reproduced.

The relations between the $\mathrm{SU}(3)$ reduced matrix elements of Ref.[6] and the quarkdiagram amplitudes are²

$$
\begin{aligned}
a & =0, \quad b=-\frac{1}{4}\left(\mathcal{C}_{A}^{\prime}+\mathcal{C}_{2 A}\right)-\frac{1}{4 \sqrt{3}}\left(\mathcal{C}_{S}^{\prime}+\mathcal{C}_{2 S}\right), \\
c & =\frac{1}{4}\left(\mathcal{A}_{A}+\mathcal{B}_{A}^{\prime}\right)-\frac{1}{2 \sqrt{3}}\left(\mathcal{C}_{S}^{\prime}+\mathcal{C}_{2 S}\right), \quad d=\frac{1}{2 \sqrt{3}}\left(\mathcal{C}_{S}^{\prime}+\mathcal{C}_{2 S}\right), \\
e & =\frac{1}{8}\left(\mathcal{A}_{A}-\mathcal{B}_{A}^{\prime}\right)+\frac{1}{4 \sqrt{3}}\left(\mathcal{C}_{S}^{\prime}-\mathcal{C}_{2 S}\right), \\
f & =-\frac{1}{8}\left(2 \mathcal{C}_{1 A}+\mathcal{C}_{A}^{\prime}-\mathcal{C}_{2 A}\right)+\frac{1}{8 \sqrt{3}}\left(\mathcal{C}_{S}^{\prime}-\mathcal{C}_{2 S}\right), \\
g & =\frac{1}{8}\left(\mathcal{A}_{A}+2 \mathcal{B}_{A}-\mathcal{B}_{A}^{\prime}\right) .
\end{aligned}
$$

At first sight, it appears that there are six independent $\mathrm{SU}(3)$ parameters, but eight different quark amplitudes. However, one may make the following redefinition (this redefinition is not unique):

$$
\begin{aligned}
& \tilde{\mathcal{A}}=\mathcal{A}_{A}-\frac{2}{\sqrt{3}} \mathcal{C}_{S}^{\prime}, \quad \tilde{\mathcal{B}}^{\prime}=\mathcal{B}_{A}^{\prime}-\frac{2}{\sqrt{3}} \mathcal{C}_{2 S}, \quad \tilde{\mathcal{C}}_{S}=\mathcal{C}_{S}^{\prime}+\mathcal{C}_{2 S}, \\
& \tilde{\mathcal{C}}^{\prime}=\mathcal{C}_{A}^{\prime}-\frac{1}{\sqrt{3}} \mathcal{C}_{S}^{\prime}+\mathcal{C}_{1 A}, \quad \tilde{\mathcal{C}}_{2}=\mathcal{C}_{2 A}-\frac{1}{\sqrt{3}} \mathcal{C}_{2 S}-\mathcal{C}_{1 A},
\end{aligned}
$$

so that the amplitudes for the decay modes in Table 2 can be expressed in terms of the six quark-diagram terms $\tilde{\mathcal{A}}, \tilde{\mathcal{B}}^{\prime}, \mathcal{B}_{A}, \tilde{\mathcal{C}}^{\prime}, \tilde{\mathcal{C}}_{2}, \tilde{\mathcal{C}}_{S}$.

Table 2.a for quark-mixing-allowed decays $B_{c}(\overline{3}) \rightarrow B(8)+M(8)$ was also previously considered by Kohara [10]. In Kohara's results there are eight quark diagrams $a_{K}, b_{K}, c_{K}$, $d_{1 K}, d_{2 K}, d_{3 K}, d_{4 K}$ and $e_{K}$. The relations between our quark diagram amplitudes and those

\footnotetext{
${ }^{1}$ Note that the reduced matrix elements $a, b, c$ and $d$ introduced in Ref.[6] are associated with the operator $O_{\overline{15}}$, which transforms as a $\overline{15}$ under flavor $\mathrm{SU}(3)$ and is symmetric in color indices and hence cannot induce a baryon-baryon transition. In other words, baryon-pole diagrams are prohibited by the operator $O_{\overline{15}}$.

${ }^{2}$ Using Table 2 and the relations (61), one can perform a cross check on the SU(3) amplitudes given in Tables I-III of Ref.[6]. For example, we find a sign error in Table III, namely the squared matrix elements for $\Xi_{c}^{0} \rightarrow \Lambda^{0} K^{0}$ should read $\frac{1}{6}|a-2 b+c+2 e-4 f-4 g|^{2}$.

${ }^{3}$ In order to avoid notation confusion with the SU(3) parameters of SS [6], we add a subscript $K$ to the Kohara's quark diagram amplitudes [10].
} 
in $[10]$ are

$$
\begin{aligned}
& a_{K}=\frac{\sqrt{6}}{8} \mathcal{A}_{A}, \quad b_{K}=\frac{\sqrt{6}}{8} \mathcal{B}_{A}^{\prime}, \quad c_{K}=\frac{\sqrt{6}}{4} \mathcal{B}_{A}, \\
& d_{1 K}=\frac{\sqrt{6}}{4} \mathcal{C}_{A}^{\prime}-\frac{1}{2 \sqrt{2}} \mathcal{C}_{S}^{\prime}, \quad d_{2 K}=\frac{1}{\sqrt{2}} \mathcal{C}_{S}^{\prime}, \\
& d_{3 K}=\frac{\sqrt{6}}{4} \mathcal{C}_{2 A}-\frac{1}{2 \sqrt{2}} \mathcal{C}_{2 S}, \quad d_{4 K}=\frac{1}{\sqrt{2}} \mathcal{C}_{2 S}, \quad e_{K}=\frac{\sqrt{6}}{4} \mathcal{C}_{1 A},
\end{aligned}
$$

which are obtained by comparing Table I of [10] with our Table 2a. As emphasized before, we consider it conceptually clearer and in practice simpler to work with the orthonormal basis of quark states. 


\section{Sextet Charmed Baryon Decays}

\section{V.a. Quark Diagram Scheme for $B_{c}(6) \rightarrow B(10)+M(8)$}

There are six independent quark-diagram amplitudes for $B_{c}(6) \rightarrow B(10)+M(8)$. The amplitudes $\mathcal{B}$ and $\mathcal{C}_{1}$ are forbidden owing to the Pati-Woo theorem. The relevant diagrams and amplitudes are exhibited in Fig. 3 and Table 3, respectively.

From Table 3 we obtain the following relations, in the absence of both SU(3) violations and final state interactions:

$$
\begin{aligned}
\left|A\left(\Omega_{c}^{0} \rightarrow \Sigma^{*+} K^{-}\right)\right|^{2} & =2\left|A\left(\Omega_{c}^{0} \rightarrow \Sigma^{* 0} \bar{K}^{0}\right)\right|^{2}, \\
\left|A\left(\Omega_{c}^{0} \rightarrow \Sigma^{* 0} \eta_{0}\right)\right|^{2} & =2\left|A\left(\Omega_{c}^{0} \rightarrow \Sigma^{* 0} \eta_{8}\right)\right|^{2}, \\
\left|A\left(\Omega_{c}^{0} \rightarrow \Sigma^{* 0} \eta_{0}\right)\right|^{2} & =\frac{1}{2} s_{1}^{2}\left|A\left(\Omega_{c}^{0} \rightarrow \Xi^{* 0} \eta_{0}\right)\right|^{2}, \\
\left|A\left(\Omega_{c}^{0} \rightarrow \Sigma^{*+} \pi^{-}\right)\right|^{2} & =s_{1}^{2}\left|A\left(\Omega_{c}^{0} \rightarrow \Sigma^{*+} K^{-}\right)\right|^{2}, \\
\left|A\left(\Omega_{c}^{0} \rightarrow \Xi^{*-} K^{+}\right)\right|^{2} & =\frac{1}{3} s_{1}^{2}\left|A\left(\Omega_{c}^{0} \rightarrow \Omega^{-} K^{+}\right)\right|^{2} .
\end{aligned}
$$

It is interesting to note that the $\Omega_{c}^{0}$ decays into $\Delta^{0} \bar{K}^{0}$ and $\Delta^{+} K^{-}$are prohibited in the quark-diagram scheme

$$
\left|A\left(\Omega_{c}^{0} \rightarrow \Delta^{0} \bar{K}^{0}\right)\right|^{2}=0, \quad\left|A\left(\Omega_{c}^{0} \rightarrow \Delta^{+} K^{-}\right)\right|^{2}=0,
$$

as the quark diagram $\mathcal{C}_{1}$ is not allowed by the Pati-Woo theorem. Consequently, the corresponding reduced matrix element $\alpha$ makes no contribution.

We note that the above quark-diagram relations except for the last one listed in (65) cannot be reproduced in the SU(3)-IR approach of SS unless the reduced matrix elements $\alpha$ and $\delta$ do not contribute. Therefore, in the SU(3) limit there are only four independent quark-diagram amplitudes or reduced matrix elements. Relations between the quark-diagram amplitudes and the symmetry parameters (see Eq. (25) of Ref.[6]) are given by

$$
\begin{array}{ll}
\mathcal{A}_{S}=\beta-2 \eta, & \mathcal{B}_{S}^{\prime}=\beta+2 \eta, \\
\mathcal{C}_{S}^{\prime}=\gamma+2 \lambda, & \mathcal{C}_{2 S}=\gamma-2 \lambda .
\end{array}
$$

\section{V.b. Quark Diagram Scheme for $B_{c}(6) \rightarrow B(8)+M(8)$}

We discuss in this section the decays of sextet charmed baryons into an octet baryon and a pseudoscalar meson. The relevant quark diagrams and amplitudes are shown in Fig. 4 and Table 4, respectively.

In the $\mathrm{SU}(3)$-symmetry approach [6], there exist no any relations between the decays of $\Omega_{c}^{0} \rightarrow B(8)+M(8)$. However, from Table 4 we obtain

$$
\left|A\left(\Omega_{c}^{0} \rightarrow n \bar{K}^{0}\right)\right|^{2}=\left|A\left(\Omega_{c}^{0} \rightarrow p K^{-}\right)\right|^{2},
$$




$$
\left|A\left(\Omega_{c}^{0} \rightarrow \Sigma^{+} K^{-}\right)\right|^{2}=2\left|A\left(\Omega_{c}^{0} \rightarrow \Sigma^{0} \bar{K}^{0}\right)\right|^{2}
$$

These relations cannot be reproduced in the $\mathrm{SU}(3)$ approach [6] unless the contributions due to the $\mathrm{SU}(3)$ parameters $a$ and $d$ vanish. Therefore, Eq. (67) will provide a good test on the quark-diagram scheme. Unfortunately, these processes are either singly or quarkmixing-doubly-suppressed. We do not expect that an encouraging experimental verification will come out soon.

The relations between quark-diagram amplitudes and $\mathrm{SU}(3)$ reduced matrix elements are found to be

$$
\begin{aligned}
& a=d=0, \quad b=-\frac{1}{2 \sqrt{3}}\left(\mathcal{A}_{S}+\mathcal{B}_{S}^{\prime}\right) \\
& c+l=\frac{1}{4}\left(\mathcal{C}_{A}^{\prime}+\mathcal{C}_{2 A}\right)-\frac{1}{4 \sqrt{3}}\left(\mathcal{C}_{S}^{\prime}+\mathcal{C}_{2 S}\right) \\
& e-l=\frac{1}{2 \sqrt{3}}\left(\mathcal{C}_{S}^{\prime}+\mathcal{C}_{2 S}\right), \quad f=\frac{1}{8}\left(\mathcal{C}_{A}^{\prime}-\mathcal{C}_{2 A}\right)-\frac{1}{8 \sqrt{3}}\left(\mathcal{C}_{S}^{\prime}-\mathcal{C}_{2 S}\right), \\
& g=\frac{1}{4 \sqrt{3}}\left(\mathcal{A}_{S}-\mathcal{B}_{S}^{\prime}\right)+\frac{1}{4 \sqrt{3}}\left(\mathcal{C}_{S}^{\prime}-\mathcal{C}_{2 S}\right), \\
& h=-\frac{1}{8}\left(\mathcal{C}_{A}^{\prime}-\mathcal{C}_{2 A}\right)+\frac{1}{8 \sqrt{3}}\left(\mathcal{C}_{S}^{\prime}-\mathcal{C}_{2 S}\right)+\frac{1}{4} \mathcal{C}_{1 A}, \\
& k=-\frac{1}{4 \sqrt{3}}\left(\mathcal{A}_{S}-\mathcal{B}_{S}^{\prime}\right)-\frac{1}{4} \mathcal{B}_{A} .
\end{aligned}
$$

Therefore, there are seven independent SU(3) parameters and quark-diagram amplitudes.

\section{Conclusions}

In this paper we have given a general and unified formulation useful for the quark-diagram scheme for baryons. Here we apply it to the two-body nonleptonic weak decays of charmed baryons and express their decay amplitudes in terms of the quark-diagram amplitudes. The effects of final-state interactions and $\mathrm{SU}(3)$ violation arising in the horizontal $W$-loop quark diagrams are included in the tables. In the absence of $\mathrm{SU}(3)$ violation and final-state interactions we have obtained many relations among various decay modes. These relations provide a framework to study these effects. Some of the relations are valid even in the presence of final-state interactions when each decay amplitude in the relation contains only a single phase shift. It will be interesting to compare all these relations with future experimental data.

Our results are consistent with those from the SU(3)-IR scheme. In addition, in the quark-diagram scheme we are able to impose the Pati-Woo theorem for weak decays and obtain more specific results than those from the SU(3)-IR scheme.

We also note that the quark-mixing-allowed decays of the antitriplet charmed baryon 
into a decuplet baryon and a pseudoscalar meson can only proceed through the $W$-exchange diagram. Hence, the experimental measurement of $\Lambda_{c}^{+} \rightarrow \Delta^{++} K^{-}$implies that the $W$ exchange mechanism plays a significant role in charmed baryon decays. 


\section{ACKNOWLEDGMENTS}

This work was supported in part by the U.S. Department of Energy and the National Science Council of Taiwan under Contract No. NSC85-2112-M-001-010. 


\section{REFERENCES}

1. For a review of charmed baryons, see J.G. Körner and H.W. Siebert, Annu. Rev. Nucl. Part. Sci. 41, 511 (1991); S.R. Klein, Int. J. Mod. Phys. A5, 1457 (1990); J.G. Körner, M. Krämer, and D. Pirjol, Prog. Part. Nucl. Phys. 33, 787 (1994).

2. Particle Data Group, Phys. Rev. D50, 1173 (1994).

3. J.G. Körner, G. Kramer, and J. Willrodt, Phys. Lett. 78B, 492 (1978); Z. Phys. C2, 117 (1979); B. Guberina, D. Tadić, and J. Trampetić, Z. Phys. C13, 251 (1982); F. Hussain and M.D. Scadron, Nuovo Cimento 79A, 248 (1984); F. Hussain and K. Khan, ibid. 88A, 213 (1985); D. Ebert and W. Kallies, Phys. Lett. 131B, 183 (1983); 148B, 502(E) (1984); Yad. Fiz. 40, 1250 (1984); Z. Phys. C29, 643 (1985); H.Y. Cheng, Z. Phys. C29, 453 (1985); Yu.L. Kalinovsky, V.N. Pervushin, G.G. Takhtamyshev, and N.A. Sarikov, Sov. J. Part. Nucl. 19, 47 (1988).

4. S. Pakvasa, S.F. Tuan, and S.P. Rosen, Phys. Rev. D42, 3746 (1990); M.P. Khanna and R.C. Verma, Z. Phys. C47, 275 (1990); G. Kaur and M.P. Khanna, Phys. Rev. D44, 182 (1991); G. Turan and J.O. Eeg, Z. Phys. C51, 599 (1991).

5. H.Y. Cheng and B. Tseng, Phys. Rev. D46, 1042 (1992); D48, 4188 (1993); Q.P. Xu and A.N. Kamal, ibid. D46, 270 (1992); J.G. Körner and M. Krämer, Z. Phys. C55, 659 (1992); T. Uppal, R.C. Verma, and M.P. Khanna, Phys. Rev. D49, 3417 (1994); P. Zenczykowski, Phys. Rev. D50, 410 (1994).

6. M.J. Savage and R.P. Springer, Phys. Rev. D42, 1527 (1990).

7. S.M. Sheikholeslami, M.P. Khanna, and R.C. Verma, Phys. Rev. D43, 170 (1991); M.P. Khanna and R.C. Verma, hep-ph/9506394.

8. L.-L. Chau, in Proceedings of the VPI Workshop on Weak Interactions, AIP Conf. Proc. No. 72, Particles and Fields, Subseries No. 23, eds. G.B. Collins, L.N. Chang, J.R. Ficence, Blacksburg, Virginia, December 3-6, 1980; Phys. Rep. 95, 1 (1983).

9. L.-L. Chau and H.Y. Cheng, Phys. Rev. Lett. 56, 1655 (1986); Phys. Rev. D36, 137 (1987); ibid. D39, 2788 (1989); Phys. Lett. 222B, 285 (1987); Mod. Phys. Lett. A4, 877 (1989).

10. Y. Kohara, Phys. Rev. D44, 2799 (1991).

11. J.C. Pati and C.H. Woo, Phys. Rev. D3, 2920 (1971); K. Miura and T. Minamikawa Prog. Theor. Phys. 38, 954 (1967); J.G. Körner, Nucl. Phys. B25, 282 (1970); Z. Phys. C43, 165 (1989). 
12. See, e.g., F.E. Close, An Introduction to Quarks and Partons (Academic Press, 1979). 


\section{FIGURE CAPTIONS}

Fig. 1. Quark diagrams for the decay $B_{c}(\overline{3}) \rightarrow B(10)+M(8)$.

Fig. 2. Quark diagrams for the decay $B_{c}(\overline{3}) \rightarrow B(8)+M(8)$.

Fig. 3. Quark diagrams for the decay $B_{c}(6) \rightarrow B(10)+M(8)$.

Fig. 4. Quark diagrams for the decay $B_{c}(6) \rightarrow B(8)+M(8)$.

\section{TABLE CAPTIONS}

Table 1a. Quark-diagram amplitudes for the quark-mixing-allowed decays of $B_{c}(\overline{3}) \rightarrow B(10)+$ $M(8)$. In Tables 1-3, SU(3) symmetry breaking is parametrized only in terms of the $\mathcal{E}$ type of quark diagrams.

Table 1b. Quark-diagram amplitudes for the quark-mixing-suppressed decays of $B_{c}(\overline{3}) \rightarrow$ $B(10)+M(8)$.

Table 1c. Quark-diagram amplitudes for the quark-mixing-doubly-suppressed decays of $B_{c}(\overline{3}) \rightarrow B(10)+M(8)$.

Table 2a. Quark-diagram amplitudes for the quark-mixing-allowed decays of $B_{c}(\overline{3}) \rightarrow B(8)+$ $M(8)$.

Table 2b. Quark-diagram amplitudes for the quark-mixing-suppressed decays of $B_{c}(\overline{3}) \rightarrow$ $B(8)+M(8)$.

Table 2c. Quark-diagram amplitudes for the quark-mixing-doubly-suppressed decays of $B_{c}(\overline{3}) \rightarrow B(8)+M(8)$.

Table 3. Quark-diagram amplitudes for $B_{c}(6) \rightarrow B(10)+M(8)$.

Table 4. Quark-diagram amplitudes for $B_{c}(6) \rightarrow B(8)+M(8)$. 

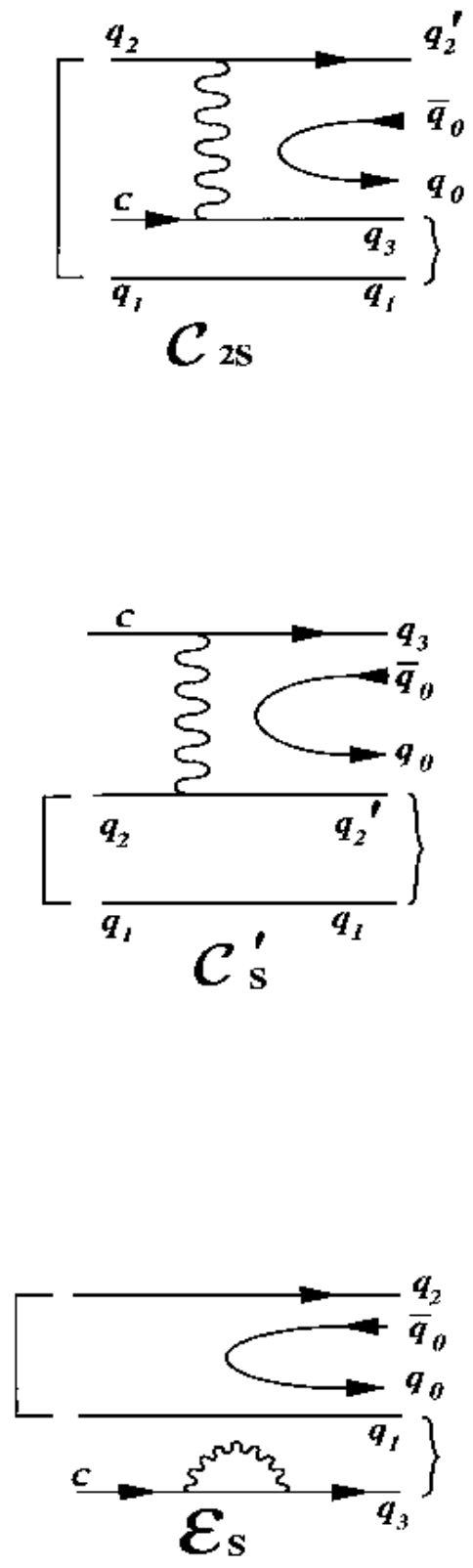

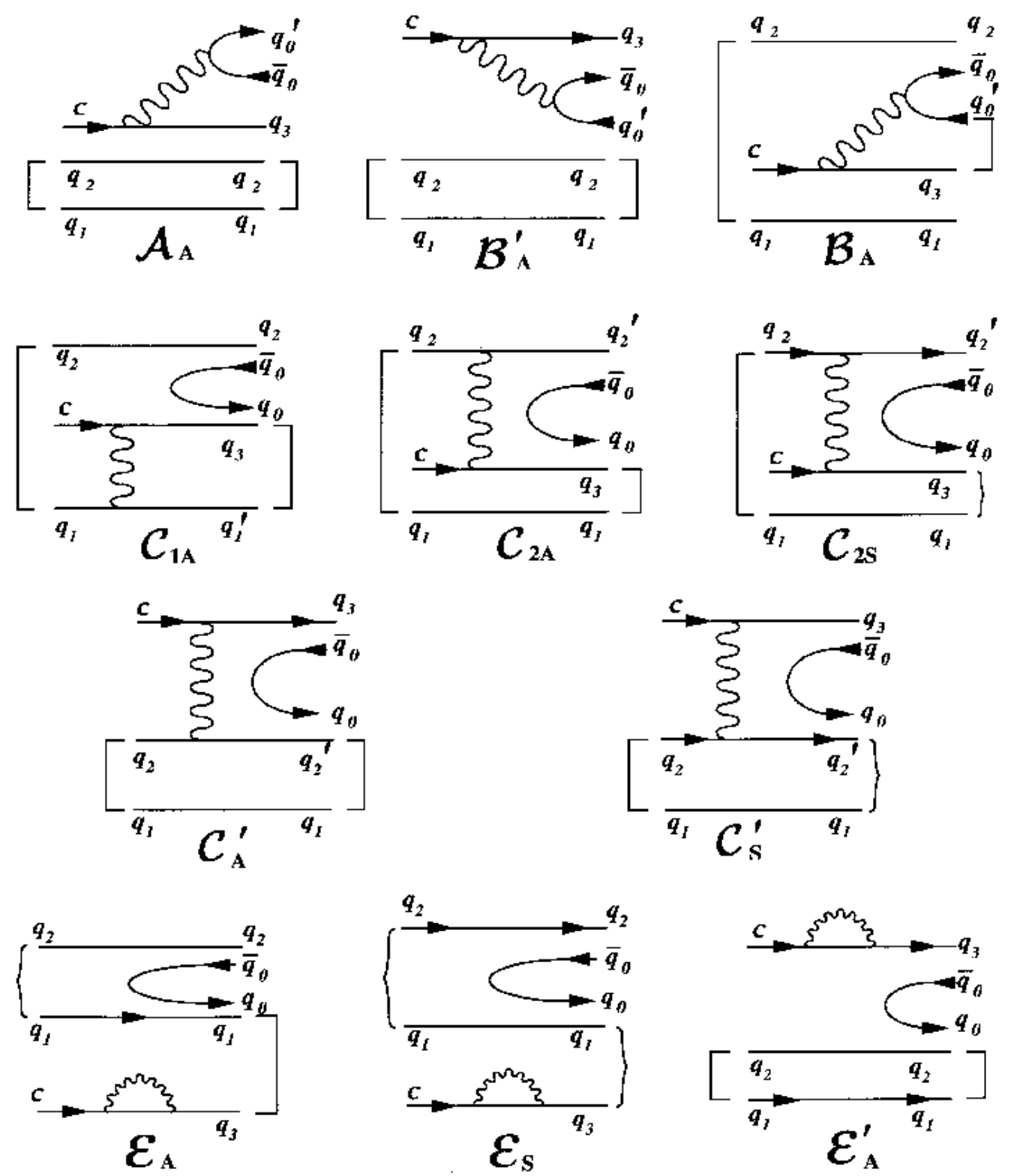


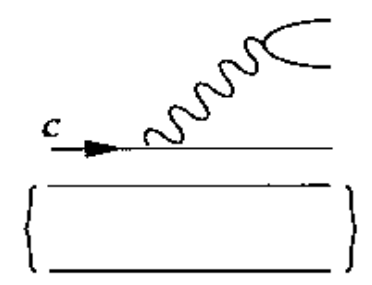

$\mathcal{A}_{\text {s }}$

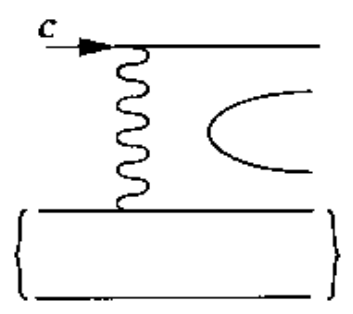

$\mathcal{C}_{\text {s }}^{\prime}$

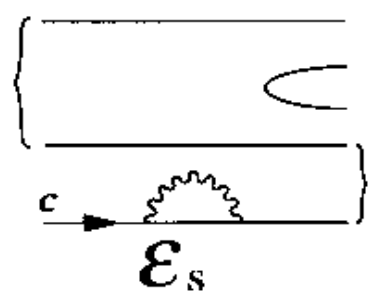

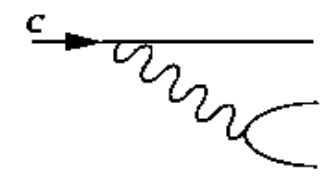

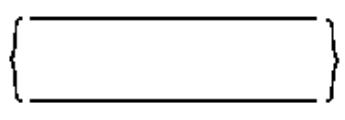

$\mathcal{B}_{\text {s }}^{\prime}$
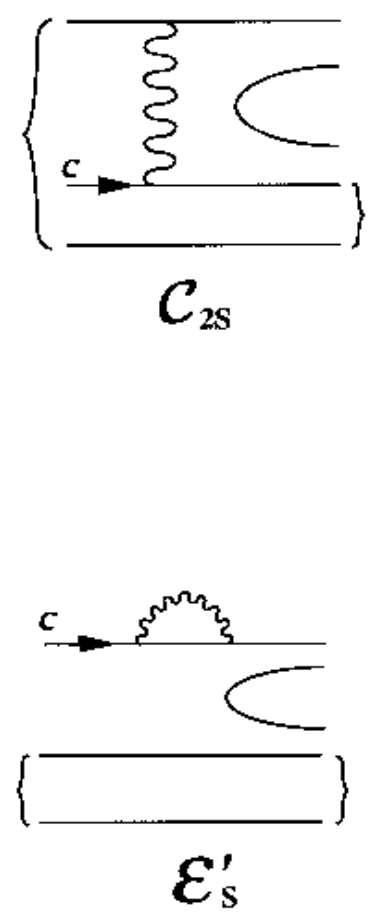


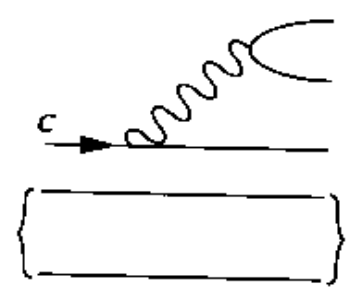

$\mathcal{A}_{\text {s }}$
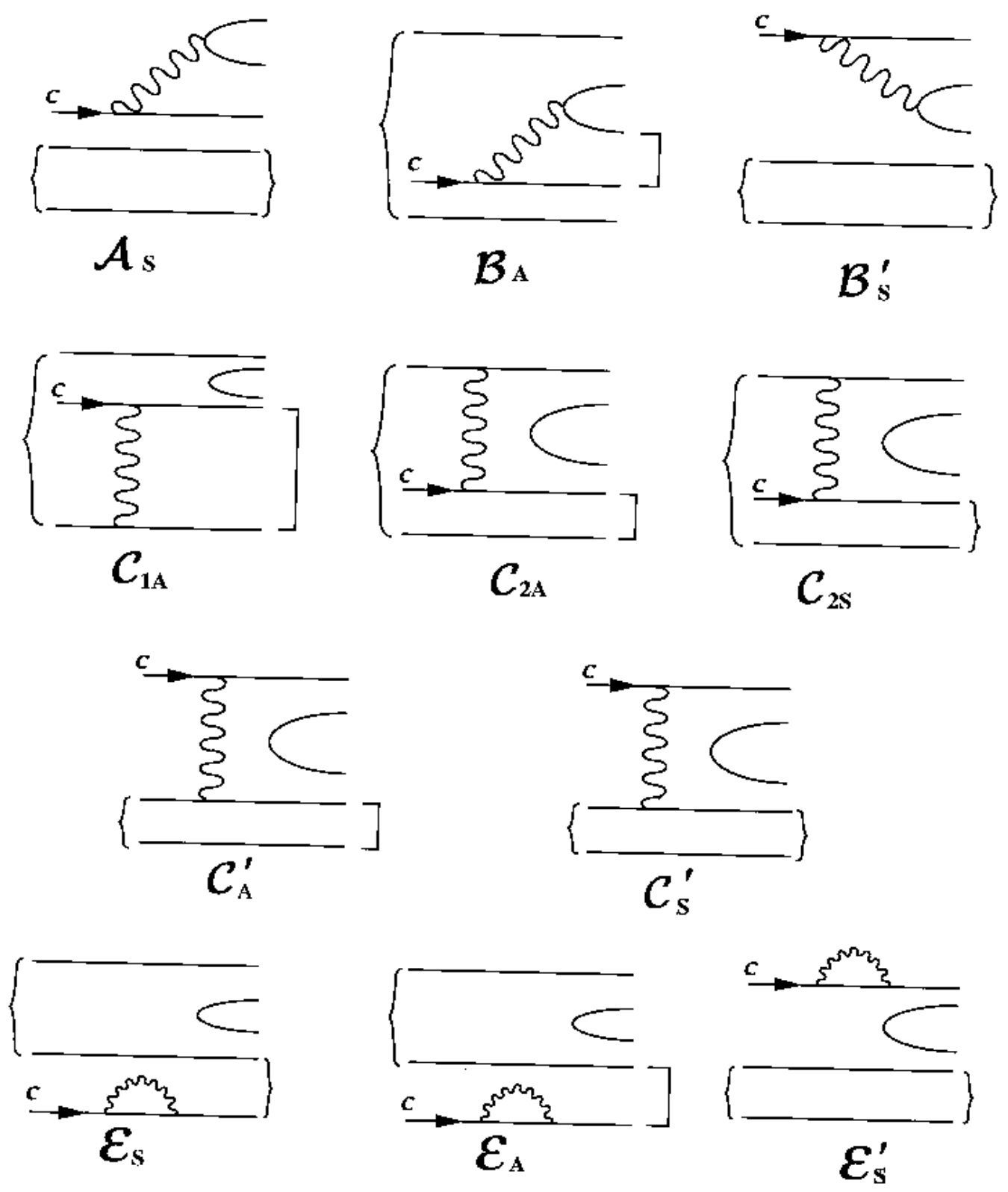